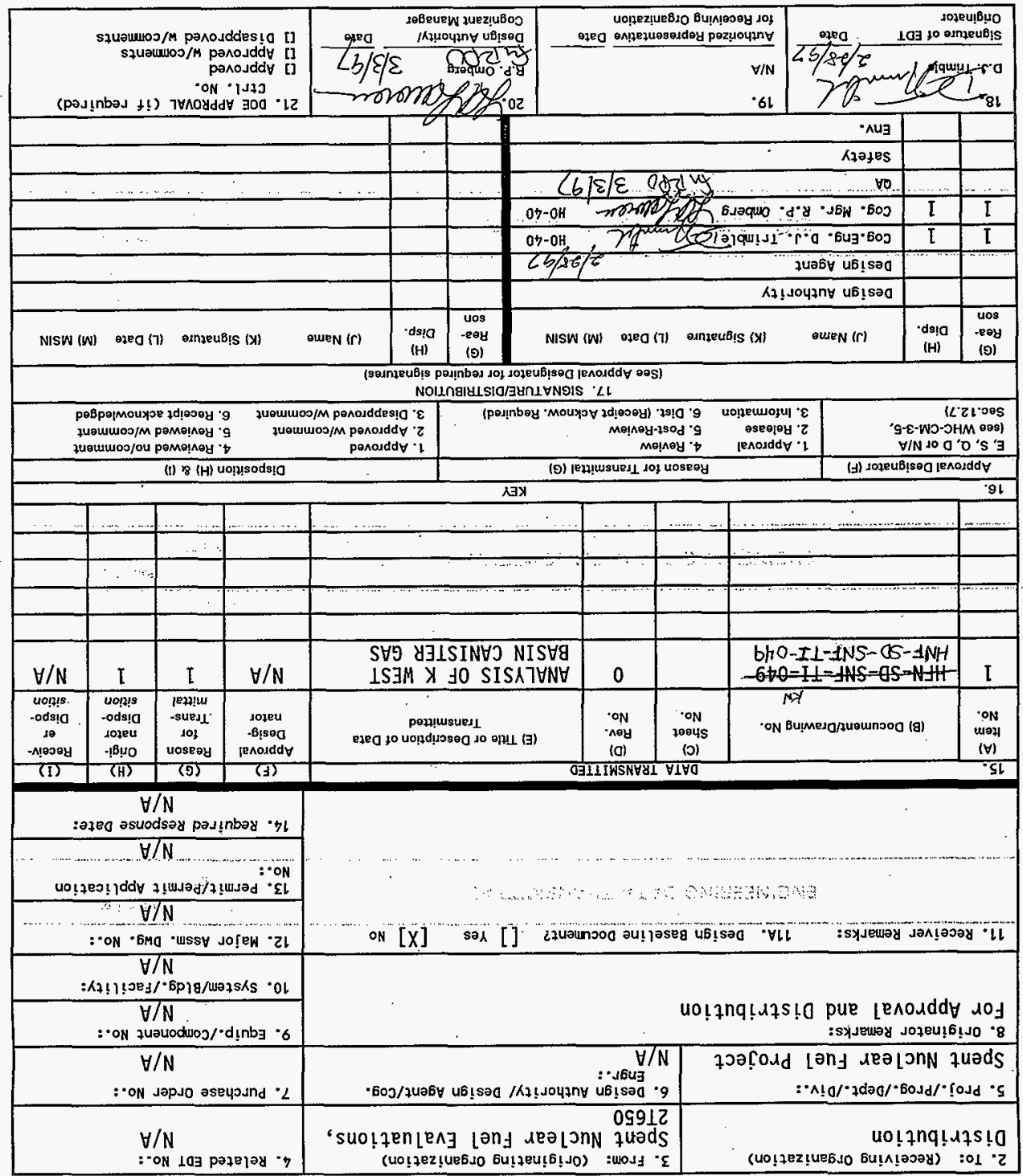

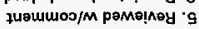
(1) 8 (H) Uọฺุsods!a 


\section{ANALYSIS OF K WEST BASIN CANISTER GAS}

D. J. Trimble

Duke Engineering \& Services Hanford, Inc., Richland, WA 99352

U.S. Department of Energy Contract DE-AC06-96RL13200

$\begin{array}{lll}\text { EDT/ECN: } & 620782 & \text { UC: } 2070 \\ \text { Org Code: } & 2 \text { T650 } & \text { Charge Code: LB022 } \\ \text { B\&R Code: } & \text { EW7040000 } & \text { Total Pages: } 87 \text { KN } 88\end{array}$

Key Words: K Basins, Spent Nuclear Fuel, Spent Fuel Storage, Characterization, $N$ Reactor Fuel, Water Analysis

Abstract: The gas in 34 barrels of 25 fuel storage canisters in $K$ West Basin was sampled in 1995 and 1996 . The samples were analyzed using gamma energy analys is and mass spectrometry. The sample data and an analysis of the data are presented. Total fission gas activity in the canisters is estimated.

TRADEMARK DISCLAIMER. Reference herein to any specific comercial product, process, or service by trade name, trademark, manufacturer, or otherwise, does not necessarily constitute or imply its endorsement, recomendation, or favoring by the united States Government or any agency thereof or its contractors or subcontractors.

Printed in the United States of America. To obtain copies of this document, contact: Document control Services, P.O. Box 950, Mailstop H6-08, Richland WA 99352, Phone (509) 372-2420;

Fax (509) 376-4989.
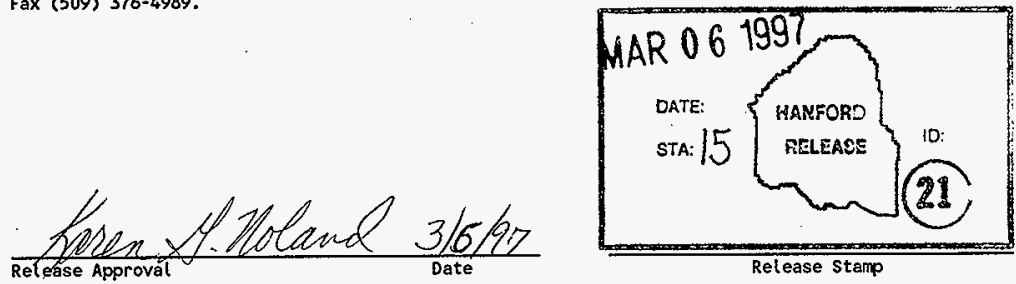

Approved for Public Release 


\section{EXECUT IVE SUMMARY}

Samples of the gas have been obtained from 34 barrels of 25 fuel storage canisters in the $K$ West Basin. Analyses of the samples revealed primarily hydrogen with some oxygen, nitrogen, and krypton-85. The hydrogen content of the gas ranged from $50 \%$ to $99 \%$ and the oxygen ranged from $0.1 \%$ to $32 \%$. Krypton-85 averaged 3,100 $\mu \mathrm{Ci} /$ barrel and measured as much as $12,700 \mu \mathrm{Ci} /$ barrel. A worst case analysis has indicated that, on average, krypton-85 should not exceed $5,500 \mu \mathrm{Ci} /$ barrel in the gas of the $\mathrm{K}$ West Basin canisters. Tritium is also expected to exist in the canister gas but should not exceed $310 \mu \mathrm{Ci} /$ barrel, on average. 
HNF-SD-SNF-TI-049, Rev. 0

\section{CONTENTS}

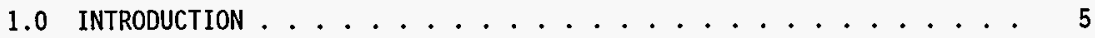

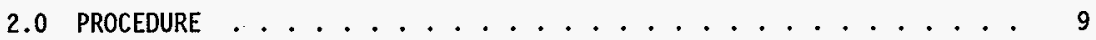

3.0 RESULTS ....................... 11

4.0 DISCUSSION ........................... 15

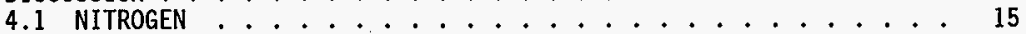

4.2 HYDROGEN ............................ 15

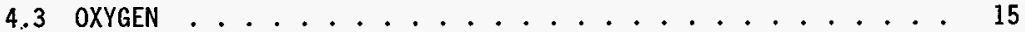

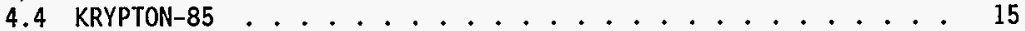

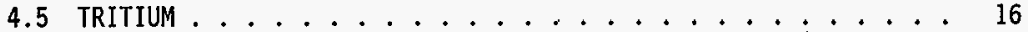

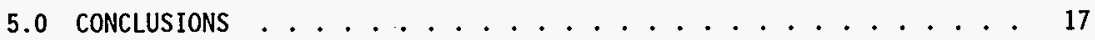

6.0 REFERENCES . . . . . . . . . . . . . . . . . . 19

APPENDIX A GAS SPECIES ANALYSIS REPORTS FOR K WEST CANISTER

SAMPLES ................. 21 
HNF-SD-SNF-TI-049, Rev. 0

\section{LIST OF FIGURES}

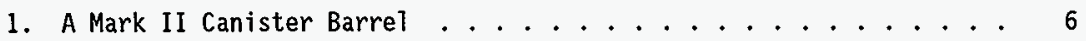

2. Oxygen and Hydrogen Data for $K$ West Basin Canister Gas . . . . . 16

\section{LIST OF TABLES}

1. K West Basin Canisters Sampled for Gas . . . . . . . . . . 7

2. Gas Sample Data from the Second (1996) Sampling Campaign . . . . 12

3. K West Basin Canister Gas Data Summary . . . . . . . . . 13

4. Summary Statistics for $K$ West Basin Canister Gas Data . . . . . 14 
HNF-SD-SNF-TI-049, Rev, 0

This page intentionally left blank. 
HNF-SD-SNF-TI-049, Rev. 0

ANALYSIS OF $K$ WEST BASIN CANISTER GAS

\subsection{INTRODUCTION}

Gas and Liquid samples have been collected from a selection of the approximately 3,820 spent fuel storage canisters in the $K$ West Basin. The samples were taken to characterize the contents of the gas and water in the canisters providing source term information for two subprojects of the Spent Nuclear Fuel Project (SNFP) (Fulton 1994): the K Basins Integrated Water Treatment System Subproject (Ba11 1996) and the K Basins Fuel Retrieval System Subproject (Waymire 1996).

The barrels of ten canisters were sampled for gas and 1iquid in 1995, and 50 canisters were sampled in a second campaign in 1996. The analysis results from the first campaign have been reported (Trimble 1995a, 1995b, 1996a, 1996b). The analysis results from the second campaign liquid samples have been documented (Trimble and Welsh 1997; Trimble 1997). This report documents the results for the gas samples from the second campaign and evaluates all gas data in terms of expected releases when opening the canisters for SNFP activities.

The fuel storage canisters consist of two closed and sealed barrels, each with a gas trap. The barrels are attached at a trunion to make a canister, but are otherwise independent (Figure 1). Each barrel contains up to seven $N$ Reactor fuel element assemblies. A gas space of nitrogen was established in the top 2.2 to 2.5 inches $(5.6$ to $6.4 \mathrm{~cm}$ ) of each barrel. Many of the fuel elements were damaged allowing the metallic uranium fuel to be corroded by the canister water. The corrosion releases fission products and generates hydrogen gas. The released gas mixes with the gas-space gas and excess gas passes through the gas trap into the basin water. The canister design does not allow canister water to be exchanged with basin water.

The canister selection and sample evaluation were in accordance with the following data quality objectives provided by Makenas 1996: (1) to help guide subsequent fuel and sludge sampling in the $\mathrm{K}$ West Basin by providing information about the condition of the fuel in specific canisters, (2) to provide information for comparison to the fuel and sludge sample examinations, and (3) to provide information to water cleanup and air permits associated with moving the fuel to dry storage. Table 1 lists the canisters sampled for gas and includes a description of each. The canister selections included both canister types, Mark I (MKI) and Mark II (MKII), and all three $\mathrm{N}$ Reactor fuel types, Mark IA (1.25\% enriched) and Mark' IV (0.95\% and $0.71 \%$ enriched).

Canisters containing documented fuel breaks and scrap fuel (chips) were also selected. In addition canisters with a range of discharge date keys, fuel burnup $\left({ }^{240} \mathrm{Pu} \%\right)$, and encapsulation dates were sampled. Also, MKII canisters containing grade "D" fuel were among the selections. Grade "D" fuel was stored for a time in the $K$ East Basin before being encapsulated at the $N$ Basin. Most $K$ West Basin fuel have resided only in the $N$ Reactor Basin before being encapsulated and shipped to $K$ West Basin. 
Figure 1. A Mark II Canister Barrel.

\section{Mk II Fuel Storage Canister}

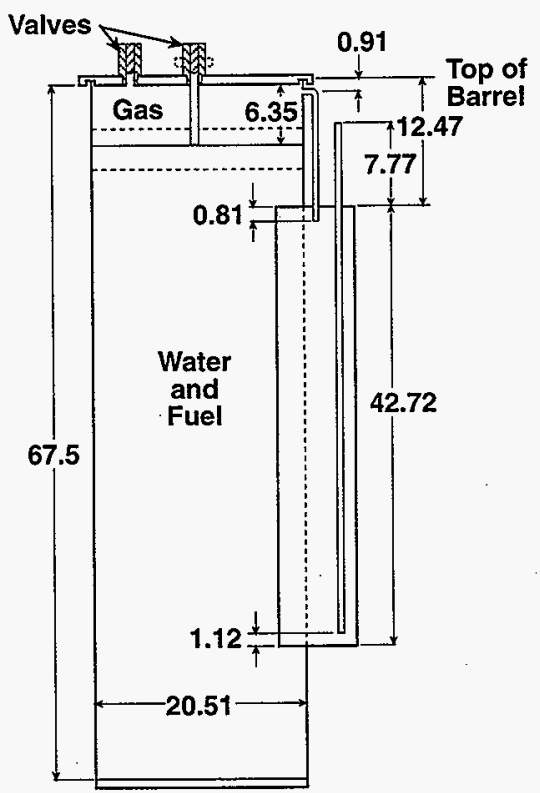

Dimensions in $\mathrm{cm}$ 
Table 1. K West Basin Canisters Sampled for Gas.

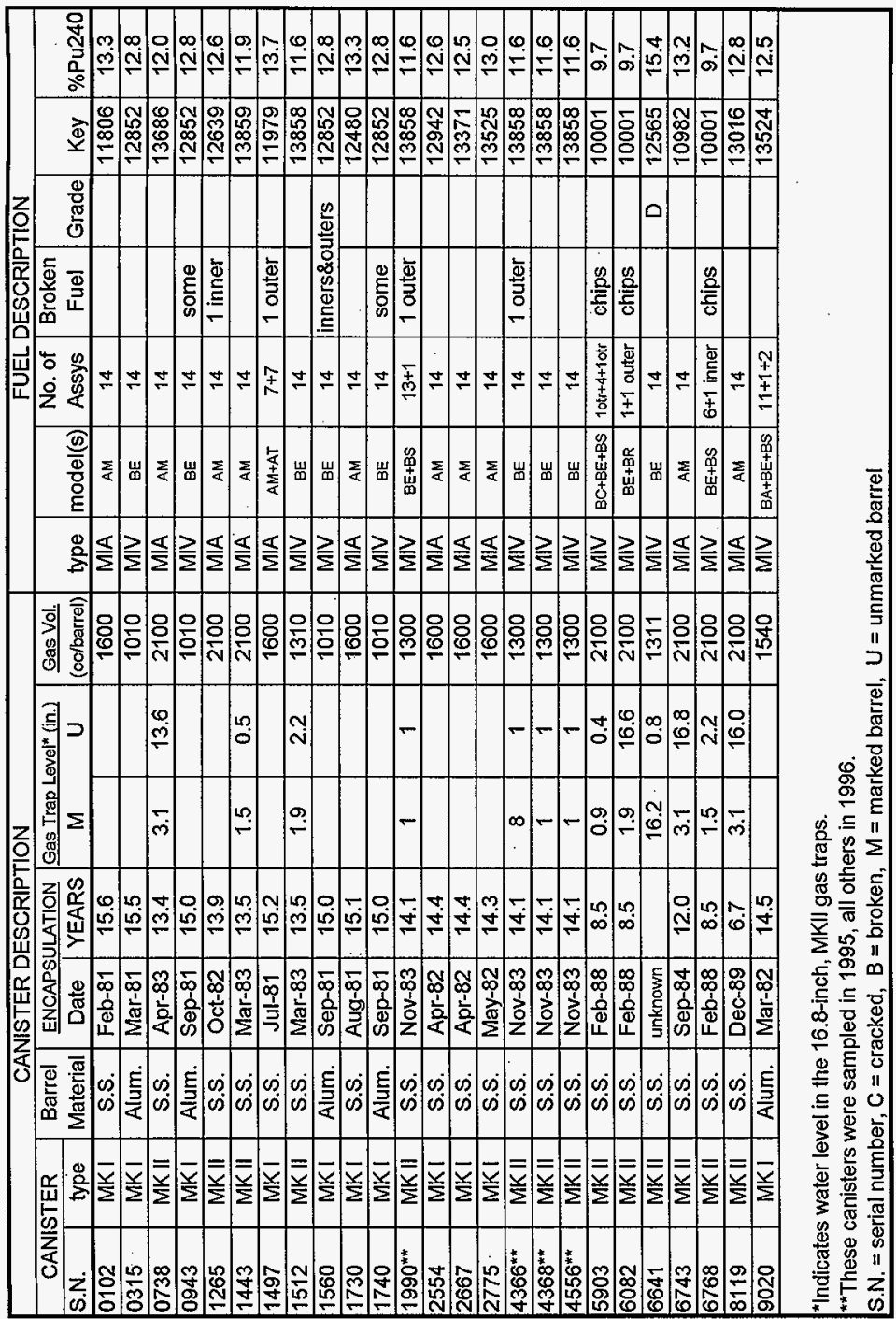


HNF-SD-SNF-TI-049, Rev. 0

This page intentionally left blank. 


\subsection{PROCEDURE}

The samples were obtained and analyzed in accordance with a sampling and analysis plan (SAP) (Harris 1996). Specially designed equipment was used to take the samples (Pitkoff 1994). Laboratory testing was performed to evaluate equipment performance characteristics and acceptability for field use (Prescott and Trimble 1996).

Gas samples were taken through the canister barrel-lid side valve before venting the gas to flood the barrel for liquid sampling. The samples were drawn into 15 and $20 \mathrm{~mL}$ vacuum vials closed with a rubber septum. The glass vials were potted in stainless steel sleeves to protect against breakage.

Before leaving the $\mathrm{K}$ Basin site and usually within 24 hours, the samples were weighed and analyzed using gamma energy analys is (GEA). The on-site GEA provided data on cesium in the liquid and krypton-85 in the gas. These analyses were performed by Special Analytical Studies of SGN Eurisys Services Corporation using their nondestructive analysis (SAS-NDA) truck. A description of the on-site GEA can be found in Trimble 1997.

The liquid samples were shipped to the Hanford 222-S Laboratory for analysis for fission products and corrosion inhibitor ions. The results of these analyses were documented in a separate report (Trimble 1997).

Gas samples were obtain from 29 barrels of 21 canisters during the 1996 campaign and five barrels of four canisters in 1995 (Trimble 1996a). They were analyzed by Pacific Northwest National Laboratory at the Hanford 325 Building using gas mass spectrography analyses. In addition sample pressure and liquid volume were measured. All gas analyses were completed within three weeks from the time of sampling.

Sample pressure was used to determine sample validity. The pressure of a gas sample should have been approximately the pressure of the canister gas. Canister pressure was about 1.4 atmosphere, due to the approximately 13 feet (4 meters) depth of the basin water at the top of the canister. An acceptable sample was at least 1.1 atmospheres. Good sample pressure was representative of the canister pressure and allowed an al iquot to be drawn through the vial septum without contamination of the aliquot from atmospheric gases. Sample gas volume at pressure was equal to the nominal volume of the sample vial (15 or $20 \mathrm{~mL}$ ) minus liquid volume. 
HNF-SD-SNF-TI-049, Rev. 0

This page intentionally left blank. 
HNF-SD-SNF-TI-049, Rev . 0

\subsection{RESULTS}

The gas analysis laboratory reports are included in Appendix A. These data are for "background corrected" results. Background corrected refers to the subtraction of residual (background) gases typically found in empty vials. Also reported was the sample pressure in atmospheres (shown as sample size, cc stp) and the volume of liquid in the sample. Sample size referred to the $1 \mathrm{cc}$ aliquot taken for analysis when expanded to standard temperature and pressure (stp).

Forty-five samples from the 1996 sampling campaign exhibited pressures above 1.1 atmospheres. The samples were from 29 barrels of 21 canisters which included multiple samples from 12 barrels. In addition one sample containing atmospheric gases was analyzed. The samples are listed in Table 2 with analysis results and a calculation of ${ }^{85} \mathrm{Kr}$ per $c c$ of sample at initial pressure. Also included in Table 2 are the sample size (volume at initial pressure) and the canister barrel from which the sample was collected.

Table 3 lists the gas data by canister barrel with averaged data for multiple samples. Also included in Table 3 are data from the first (1995) sampling campaign (Trimble 1996a). ' Summary statistics for the data are provided in Table 4. 
Table 2. Gas Sample Data from the Second (1996) Sampling Campaign.

\begin{tabular}{|c|c|c|c|c|c|c|c|c|c|}
\hline & sample & canister & hydrogen & oxygen & nitrogen & argon & sum & $\mathrm{Kr}-85$ & $\mathrm{Kr} 85$ \\
\hline SAMPLE & $\operatorname{size}^{*}(\mathrm{cc}$ & barrel & percent & percent & percent & percent & percent & $\mathrm{uC}$ & $\mathrm{uCi} / \mathrm{cc}$ \\
\hline E33 & 14.6 & $0102 \mathrm{M}$ & 83.7 & 11.1 & 5 & 0.071 & 99.87 & 14.4 & 0.99 \\
\hline E34 & 11.2 & $0102 U$ & 81.4 & 12.55 & 5.9 & 0.104 & 99.90 & 7.31 & 0.65 \\
\hline$E 20$ & 14.9 & $0315 \mathrm{M}$ & 96.4 & 1.74 & 1.78 & 0.042 & 99.96 & \begin{tabular}{|c|}
3.95 \\
\end{tabular} & 0.27 \\
\hline$E 22$ & 14.4 & $0315 \mathrm{U}$ & $\begin{array}{l}74.4 \\
\end{array}$ & 21.4 & 4.04 & 0.069 & 99.91 & 4.6 & 0.32 \\
\hline$E 27$ & 14.2 & $0738 \mathrm{M}$ & 61.8 & 26.1 & 11.9 & 0.129 & 99.93 & 9.83 & 0.69 \\
\hline E28 & 14.0 & $0738 \mathrm{U}$ & 51.6 & 3.6 & 44.4 & 0.29 & 99.89 & 29 & 2.07 \\
\hline$E 29$ & 14.0 & 07380 & 50.2 & 1.23 & 48.2 & 0.31 & 99.94 & 29.5 & 2.11 \\
\hline$\overline{D 071}$ & 19.0 & 0943M & 92.0 & 2.73 & 5.1 & 0.081 & 99.91 & 23.4 & 1.23 \\
\hline$E 38$ & 14.9 & $1265 \mathrm{M}$ & 55.0 & 0.63 & 44 & 0.206 & 99.84 & 38 & 2.55 \\
\hline E36 & 14.9 & $1265 \mathrm{U}$ & 74.2 & 1.59 & 24 & 0.155 & 99.95 & 43.5 & 2.92 \\
\hline E37 & 14.6 & $1265 U$ & 71.8 & 0.77 & 27.1 & 0.181 & 99.85 & 46.3 & 3.17 \\
\hline D029 & 15.1 & $1443 \mathrm{M}$ & 49.3 & 2.42 & 47.8 & $\begin{array}{ll}0.362 \\
\end{array}$ & 99.88 & 18 & 1.19 \\
\hline$\overline{\mathrm{D} 030}$ & 17.2 & $1443 \mathrm{M}$ & 51.0 & 1.71 & 46.9 & 0.337 & 99.95 & 21 & 1.22 \\
\hline$\overline{E 39}$ & 13.6 & $1497 \mathrm{M}$ & 82.1 & 4.05 & 13.6 & 0.135 & 99.89 & 30.5 & 2.24 \\
\hline D165 & 19.5 & 1497M & 80.4 & 5.35 & 13.9 & 0.173 & 99.82 & 58 & 2.97 \\
\hline$E 40$ & 14.0 & $1497 \mathrm{U}$ & 77.8 & 4.54 & 17.3 & 0.236 & 99.88 & 32.3 & 2.31 \\
\hline$\overline{D 166}$ & 18.6 & $1497 \mathrm{U}$ & 84.1 & 3.28 & 12.3 & 0.164 & 99.84 & 50 & 2.69 \\
\hline$\overline{D 101}$ & 16.5 & $1512 U$ & 89.5 & 1.53 & 8.8 & 0.148 & 99.98 & 30 & 1.82 \\
\hline E09 & 13.1 & $1560 \mathrm{M}$ & 95.1 & 0.925 & 3.85 & 0.071 & 99.95 & 30.4 & 2.32 \\
\hline E11 & 15.0 & $\overline{1560 \mathrm{M}}$ & 97.2 & 0.49 & 2.17 & 0.047 & 99.91 & 33.9 & 2.26 \\
\hline$\overline{D 102}$ & 19.9 & $1560 \mathrm{M}$ & 93.8 & 0.94 & 5.1 & 0.07 & 99.91 & 52 & 2.61 \\
\hline$E 12$ & 15.0 & $1560 \mathrm{U}$ & 99.9 & $\overline{0}$ & 0 & 0.006 & 99.91 & 60 & 4.00 \\
\hline D103 & 19.5 & $1560 \mathrm{U}$ & 94.0 & 1.17 & 4.63 & 0.065 & 99.87 & 85 & 4.36 \\
\hline$\overline{D 178}$ & 19.1 & $1730 \mathrm{U}$ & 55.7 & 2.04 & 41.9 & 0.253 & 99.97 & 74 & 3.87 \\
\hline E14 & 13.5 & $1740 \mathrm{M}$ & 58.2 & 28.1 & 13.5 & 0.169 & 99.97 & 0 & 0.00 \\
\hline E16 & 14.0 & 17400 & 88.4 & 6.5 & 5 & 0.081 & 99.98 & 7.56 & 0.54 \\
\hline$\overline{D 026 n c}$ & 15.5 & $2554 \mathrm{M}$ & 45.0 & 2.96 & 51 & 0.366 & 99.33 & 37 & 2.39 \\
\hline D027 & 18.9 & $2554 \mathrm{M}$ & 49.0 & 1.46 & 49.2 & 0.307 & 99.97 & 38 & 2.01 \\
\hline E06 & 11.5 & $2667 \mathrm{M}$ & 84.5 & 3.445 & 11.85 & 0.167 & 99.91 & 29.6 & 2.57 \\
\hline D181 & 17.6 & $2667 \mathrm{M}$ & 77.2 & 4.11 & 18.4 & 0.227 & 99.94 & 50 & 2.84 \\
\hline \begin{tabular}{|l|l|}
$\mathrm{D} 182$ \\
\end{tabular} & 19.3 & $2667 \mathrm{M}$ & 90.6 & 1.63 & 7.6 & \begin{tabular}{|c|}
0.113 \\
\end{tabular} & 99.88 & 58 & 3.01 \\
\hline$E 41$ & 12.4 & $2775 \mathrm{M}$ & 56.4 & 2.66 & 40.5 & 0.321 & 99.88 & 15.8 & 1.27 \\
\hline$E 42$ & 13.8 & $2775 \mathrm{U}$ & 81.4 & 0.99 & 17.4 & 0.167 & 99.96 & 40.5 & 2.93 \\
\hline$\overline{D 014}$ & 15.3 & \begin{tabular}{|l|l|}
$5903 \mathrm{U}$ \\
\end{tabular} & 86.9 & 2.47 & 10.3 & 0.147 & 99.82 & 10 & 0.65 \\
\hline D097 & 19.5 & $5903 \mathrm{U}$ & 94.0 & 1.15 & 4.69 & 0.07 & 99.91 & 21 & 1.08 \\
\hline E18 & 15.0 & $6082 \mathrm{M}$ & 97.4 & 0.61 & 1.96 & 0.041 & 99.96 & 14.5 & 0.97 \\
\hline E25 & 15.0 & 66410 & 65.5 & 33.1 & 1.38 & 0.033 & 100.01 & 1.33 & 0.09 \\
\hline$E 26$ & 14.9 & $6641 \mathrm{U}$ & 57.6 & 31.7 & 10.5 & 0.142 & 99.94 & 1.28 & 0.09 \\
\hline E13 & 14.5 & $6743 \mathrm{M}$ & 81.0 & 1.14 & 17.6 & 0.152 & 99.89 & 38 & 2.62 \\
\hline D175 & 15.5 & $6768 \mathrm{U}$ & 82.4 & 2.57 & 14.5 & 0.188 & 99.66 & 94 & 6.06 \\
\hline D034 & 17.6 & $8119 \mathrm{M}$ & 60.2 & 29.15 & 10.45 & 0.1475 & 99.95 & 8.6 & 0.49 \\
\hline E01 & 13.2 & $9020 \mathrm{M}$ & 89.3 & 1.495 & 8.9 & 0.149 & 99.84 & 11.2 & 0.85 \\
\hline D019 & 16.2 & $9020 \mathrm{M}$ & 87.0 & 2.19 & 10.5 & 0.165 & 99.86 & 14.6 & 0.90 \\
\hline D020 & 18.8 & $9020 \mathrm{M}$ & 89.2 & 1.58 & 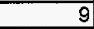 & 0.132 & 99.91 & 12.3 & 0.65 \\
\hline D021 & 18.5 & $9020 \mathrm{M}$ & 78.7 & 3.68 & 17.3 & 0.222 & 99.90 & 11 & 0.59 \\
\hline$E 49^{* *}$ & 15.0 & BLANK & 0.02 & 21.3 & 77.7 & 0.940 & 99.96 & 0.00 & 0.00 \\
\hline \multicolumn{10}{|c|}{ ras vial size minus measure } \\
\hline \multicolumn{10}{|c|}{ and $D X X X$ samples were in $20 \mathrm{~mL}$ vials } \\
\hline **This sa & mple was & atmosph & eric gas (a) & & & & & & \\
\hline
\end{tabular}


Table 3. K West Basin Canister Gas Data Summary.

\begin{tabular}{|c|c|c|c|c|c|c|}
\hline canister & hydrogen & oxygen & nitrogen & Kr85 & Kr85 & Cs-137** \\
\hline barrel & percent & percent & percent & uCi/cc & mCi/barrel & mCi/barrel \\
\hline $0102 \mathrm{M}$ & 83.7 & 11.1 & 5.0 & 0.99 & 1.58 & 623 \\
\hline $0102 \mathrm{U}$ & 81.4 & 12.6 & 5.9 & 0.65 & 1.04 & 395 \\
\hline $0315 \mathrm{M}$ & 96.4 & 1.7 & 1.8 & 0.27 & 0.27 & 119 \\
\hline $0315 \mathrm{U}$ & 74.4 & 21.4 & 4.0 & 0.32 & 0.32 & 142 \\
\hline $0738 \mathrm{M}$ & 61.8 & 26.1 & 11.9 & 0.69 & 1.45 & 175 \\
\hline $0738 \mathrm{U}$ & 50.9 & 2.4 & 46.3 & 2.09 & 4.38 & 177 \\
\hline $0943 M$ & 92.0 & 2.7 & 5.1 & 1.23 & 1.25 & 494 \\
\hline $1265 \mathrm{M}$ & 55.0 & 0.6 & 44.0 & 2.55 & 5.35 & 402 \\
\hline $1265 \mathrm{U}$ & 73.0 & 1.2 & 25.6 & 3.05 & 6.39 & 703 \\
\hline $1443 \mathrm{M}$ & 50.2 & 2.1 & 47.4 & 1.21 & 2.53 & no data \\
\hline $1497 \mathrm{M}$ & 81.3 & 4.7 & 13.8 & 2.61 & 4.17 & 358 \\
\hline $1497 \mathrm{U}$ & 81.0 & 3.9 & 14.8 & 2.50 & 4.00 & 4046 \\
\hline $1512 \mathrm{U}$ & 89.5 & 1.5 & 8.8 & 1.82 & 2.38 & 246 \\
\hline $1560 \mathrm{M}$ & 95.4 & 0.8 & 3.7 & 2.40 & 2.42 & 179 \\
\hline $1560 U$ & 97.0 & 0.6 & 2.3 & 4.28 & 4.32 & 1242 \\
\hline $1730 \mathrm{U}$ & 55.7 & 2.0 & 41.9 & 3.87 & 6.20 & no data \\
\hline $1740 \mathrm{M}$ & 58.2 & 28.1 & 13.5 & 0.00 & 0.00 & 40 \\
\hline $1740 \mathrm{U}$ & 88.4 & 6.5 & 5.0 & 0.54 & 0.55 & 366 \\
\hline $1990 \mathrm{M}^{*}$ & 72.0 & 0.14 & 27.6 & 3.53 & 4.63 & 170 \\
\hline $2554 \mathrm{M}$ & 47.0 & 2.2 & 50.1 & 2.26 & 3.62 & no data \\
\hline $2667 M$ & 84.1 & 3.1 & 12.6 & 2.81 & 4.49 & 3558 \\
\hline $2775 \mathrm{M}$ & 56.4 & 2.7 & 40.5 & 1.27 & 2.04 & 101 \\
\hline $2775 \mathrm{U}$ & 81.4 & 1.0 & 17.4 & 2.93 & 4.70 & 340 \\
\hline $4366 \mathrm{M}^{\star}$ & 65.7 & 0.56 & 33.5 & 1.80 & 2.36 & no data \\
\hline $4368 \mathrm{M}^{*}$ & 86.4 & 10.8 & 2.59 & 1.36 & 1.78 & 210 \\
\hline $4368 \mathrm{U}^{*}$ & 98.6 & 0.27 & 1.06 & 1.79 & 2.34 & 340 \\
\hline $4556 \mathrm{M}^{\star}$ & 94.5 & 0.22 & 5.1 & 3.00 & 3.93 & 200 \\
\hline $5903 \mathrm{U}$ & 90.5 & 1.8 & 7.5 & 0.87 & 1.81 & 3247 \\
\hline $6082 M$ & 97.4 & 0.6 & 2.0 & 0.97 & 2.03 & 1232 \\
\hline $6641 U$ & 61.6 & 32.4 & 5.9 & 0.09 & 0.11 & 86 \\
\hline $6743 \mathrm{M}$ & 81.0 & 1.1 & 17.6 & 3.66 & 7.67 & 807 \\
\hline $6768 \mathrm{U}$ & 82.4 & 2.6 & 14.5 & 6.06 & 12.72 & no data \\
\hline $8119 \mathrm{M}$ & 60.2 & 29.2 & 10.5 & 0.49 & 1.02 & 174 \\
\hline $9020 \mathrm{M}$ & 86.1 & 2.2 & 11.4 & 0.82 & 1.26 & no data \\
\hline
\end{tabular}


Table 4. Summary Statistics for $K$ West Basin Canister Gas Data.

\begin{tabular}{|c|c|c|c|c|c|c|c|c|}
\hline \multirow{2}{*}{$\frac{\text { FUEL }}{\text { TYPE }}$} & \multirow[b]{2}{*}{ statistic } & hydrogen & oxygen & nitrogen & $\mathrm{Kr} 85^{*}$ & Kr85 & $\mathrm{Kr}-\mathrm{max}^{\star \star \star}$ & Cs-137 \\
\hline & & \multirow{2}{*}{\multicolumn{2}{|c|}{$\begin{array}{ll}\text { percent } & \text { percent } \\
\end{array}$}} & \multirow[t]{2}{*}{ percent } & \multirow[t]{2}{*}{$\mathrm{uCi} / \mathrm{cc}$} & \multirow[t]{2}{*}{ mCi/barre } & \multirow[t]{2}{*}{ mCi/barrel } & \multirow{2}{*}{ mCi/barrel } \\
\hline & & & & & & & & \\
\hline MKIA & $\mathrm{MEAN}=$ & 67.74 & 6.62 & 25.32 & 2.10 & 3.79 & 5.78 & 912 \\
\hline$\overline{M K I A}$ & MEDIAN $=$ & 67.74 & 2.66 & 17.60 & 2.26 & 4.00 & 5.36 & 399 \\
\hline MK IA & STD DEV= & 14.15 & 8.90 & 16.56 & 1.09 & 2.03 & 3.15 & 1304 \\
\hline MKIA & MAX $=$ & 84.08 & 29.15 & 50.10 & 3.87 & 7.67 & 11.13 & 4046 \\
\hline MKIA & $\mathrm{MIN}=$ & 47.00 & 0.63 & 5.00 & 0.49 & 1.02 & 1.28 & 101 \\
\hline MK IA & $\mathrm{N}=$ & 16 & 16 & 16 & 16 & 16 & 16 & 16 \\
\hline & & 原 & & & 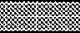 & . & . & 8 \\
\hline MK IV & MEAN= & 84.79 & 6.39 & 8.63 & 1.73 & 2.47 & 2.94 & 554 \\
\hline MK IV & MEDIAN= & 88.95 & 1.78 & 5.10 & 1.30 & 1.92 & 2.14 & 210 \\
\hline MKIV & STD DEV $=$ & 12.95 & 10.15 & 8.95 & 1.62 & 2.91 & 3.58 & 834 \\
\hline MK IV & MAX $=$ & 98.60 & 32.40 & 33.50 & 6.06 & 12.72 & 15.43 & 3247 \\
\hline MK IV & $\mathrm{MIN}=$ & 58.20 & 0.14 & 1.06 & 0.00 & 0.00 & 0.00 & 40 \\
\hline MKIV & $\mathrm{N}=$ & 18 & 18 & 18 & 18 & 18 & 18 & 18 \\
\hline 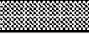 & (n) & 8. & & & & & & Fin \\
\hline all & MEAN $=$ & 76.77 & 6.50 & 16.49 & 1.90 & 3.09 & 4.27 & 720 \\
\hline all & MEDIAN= & 81.30 & 2.22 & 11.66 & 1.79 & 2.37 & 3.24 & 340 \\
\hline all & STD DEV $=$ & 15.87 & 9.44 & 15.41 & 1.38 & 2.59 & 3.63 & 1072 \\
\hline all & $\mathrm{MAX}=$ & 98.60 & 32.40 & 50.10 & 6.06 & 12.72 & 15.43 & 4046 \\
\hline all & $\mathrm{MIN}=$ & 47.00 & 0.14 & 1.06 & 0.00 & 0.00 & 0.00 & 40 \\
\hline all & $\mathrm{N}=$ & 34 & 34 & 34 & 34 & 34 & 34 & 34 \\
\hline & 8. & & & & & & & \\
\hline $\begin{array}{l}\text { *At samr } \\
{ }^{*} \mathrm{Kr}-85 \\
\mathrm{~N}=\mathrm{Num}\end{array}$ & $\begin{array}{l}\text { pressure } \\
\text { usted to } 10 \\
\text { r of observ }\end{array}$ & $\begin{array}{l}\text { hydr } \\
\text { ons }\end{array}$ & & & & & & \\
\hline
\end{tabular}




\subsection{DISCUSSION}

The following discussion summarizes the gas data from samples taken in 1995 and 1996 (first and second gas/liquid sampling campaigns) from K West Basin canisters.

\subsection{NITROGEN}

The canister gas was initially pure nitrogen. Over time much of this gas has been displaced by hydrogen and smaller amounts of oxygen. Nitrogen in the sampled barrels ranged from $1 \%$ to $50 \%$ with a mean $16 \%$.

\subsection{HYDROGEN}

The samples were primarily hydrogen, ranging from $47 \%$ to $99 \%$ with a mean 77\%. The hydrogen source is the canister water. Mechanisms that can generate gas from the water include radiolytic decomposition and release by uranium corrosion (Trimble 1996a).

\subsection{OXYGEN}

0xygen in the sample barrels ranged from $0.1 \%$ to $32 \%$ with a mean $6.5 \%$. Four of the 25 barrels sampled contained $26 \%$ to $32 \%$ oxygen with $58 \%$ to $62 \%$. hydrogen (Figure 2). This ratio of 0.5 for oxygen to hydrogen was not exceeded in any sample and was typically much lower. The ratio 0.5 indicates radiolytic decomposition. Ratios less than 0.5 suggests gas generation from a combination of corrosion hydrogen and radiolysis.

\subsection{KRYPTON-85}

The gas typically contained smal1 amounts of ${ }^{85} \mathrm{Kr}$ activity averaging $1.9 \mu \mathrm{Ci} / \mathrm{CC}$ and with a maximum $6.1 \mu \mathrm{Ci} / \mathrm{cc}$ (at canister pressure). Total ${ }^{85} \mathrm{Kr}$ per barrel averaged 3,100 $\mu \mathrm{Ci}$ with maximum $12,700 \mu \mathrm{Ci}$.

It is assumed that ${ }^{85} \mathrm{Kr}$ is released from the canister fuel as the result of corrosion of the fuel. A fission-gas release model assumed all gas space nitrogen was replaced by corrosion hydrogen providing a worst case prediction of ${ }^{85} \mathrm{Kr}$ in the gas (Trimble 1996b). This model predicted 5,500 $\mu \mathrm{Ci} / \mathrm{barrel}$ $(11,000 \mu \mathrm{Ci} /$ canister $)$ as an average for all $\mathrm{K}$ West Basin canisters (Trimble 1996), which is $180 \%$ of the sample mean.

The gas in canisters storing Mark IV fuel could contain an average $3,600 \mu \mathrm{C} / \mathrm{barrel}$ of ${ }^{85} \mathrm{Kr}$ as predicted by the fission-gas release model. Similarly, canisters with Mark IA fuel could average $7,000 \mu \mathrm{Ci} / \mathrm{barrel}$ (Trimble 1996b). Since the mode7 assumes $100 \%$ corrosion hydrogen in the 
gas space, a comparison of these predictions requires that the data be adjusted to $100 \%$ hydrogen, i.e., $\mu \mathrm{Ci}$ per $c c$ of hydrogen. Using this transformation and multiplying by the barrel gas space volume, the samples from barrels containing Mark IV fuel averaged 2,950 $\mu \mathrm{Ci} /$ barre1, and those from barrels with Mark IA fuel averaged $5,360 \mu \mathrm{Ci} /$ barrel (Table 4 ). In both cases the sample data averages were less than the predicted values by no more than $25 \%$.

\subsection{TRITIUM}

Tritium was not an analyte of the gas samples but is a fission gas that may be released by fuel corrosion. The fission-gas release model discussed in Section 4.4 predicts that $310 \mu \mathrm{Ci}$ /barrel of tritium could be released to the gas of an average canister in $K$ West Basin (Trimble 1996b). This should be worst case for two reasons. First, significant amounts of tritium are found in the canister water (Trimble 1996b, 1997). The amount of tritium in the water appears to have been about one-third of that in the corroded fuel (Trimble 1996b), the remainder of which is expected to be in the gas. Secondly, not all of the gas space nitrogen has been displaced with hydrogen, and it is the corrosion hydrogen that carries fission gases into the canister gas.

Figure 2. 0xygen and Hydrogen Data for $K$ West Basin Canister Gas.

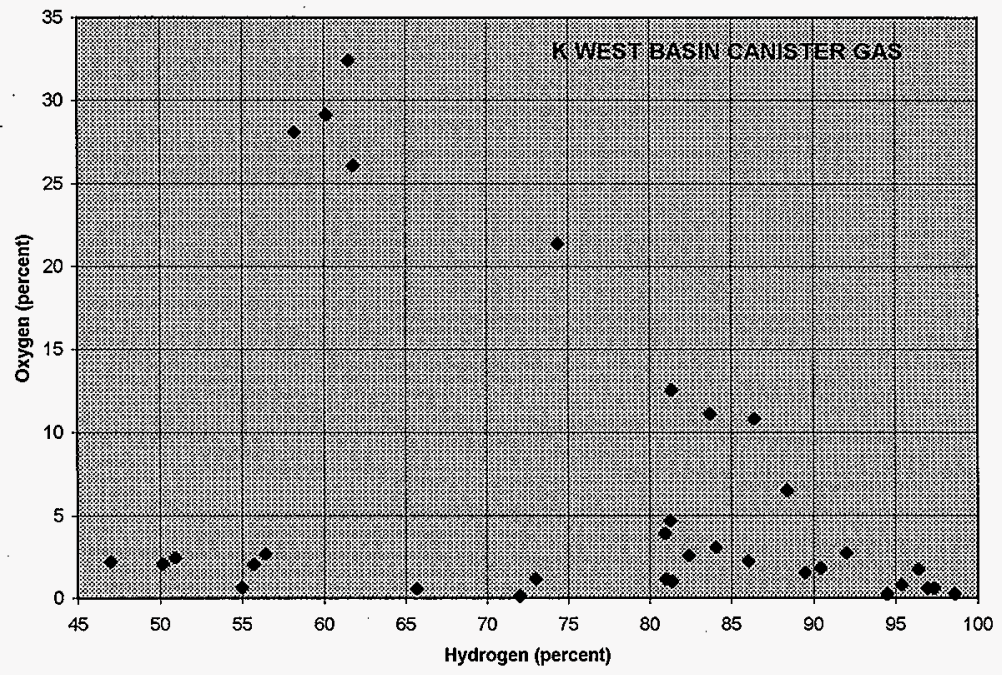




\subsection{CONCLUSIONS}

1. The $K$ West Basin canister gas consists of primarily hydrogen with some nitrogen and oxygen. Nitrogen is the original canister gas added during encapsulation. The hydrogen and oxygen are from the canister water. Radiolytic decomposition of the water produces: hydrogen and oxygen gases, and water corrosion of the stored fuel generates hydrogen. In most canisters, the corrosion hydrogen has predominated over the radiolysis gases.

2. The canister gas contains fission gases. Krypton-85 averaged $3,100 \mu \mathrm{Ci} /$ barre $1(6,200 \mu \mathrm{Ci} /$ canister $)$ compared to a worst case prediction of $11,000 \mu \mathrm{Ci} / \mathrm{canister}$. Krypton-85 in the gas should not exceed $11,200 \mu \mathrm{Ci} /$ canister, on average.

3. Tritium in the gas should not exceed $620 \mu \mathrm{Ci} /$ canister, on average. 
HNF-SD-SNF-TI-049, Rev. 0

This page intentionally left blank. 


\subsection{REFERENCES}

Ball, D. E., M. A. Knight, and T. R. Pauly, 1996, Preliminary Design Report for the $K$ Basins Integrated Water Treatment System, WHC-SD-SNF-RPT-012, Rev. 0, Westinghouse Hanford Company, Richland, Washington.

Fulton, J. C., 1994, Hanford Spent Nuclear Fuel Project Recommended Path Forward, Vo 7. 1, WHC-EP-0830, Rev. 0, Westinghouse Hanford Company, Richland, Washington.

Makenas, B. J., 1995, Data Quality Objectives for Gas and Liquid Samples from Sealed K Basin Canister, WHC-SD-SNF-DQ0-003, Rev. 0, Westinghouse Hanford Company, Richland, Washington.

Pitkoff, C. C., 1994, Gas and Liquid Samples for Closed Canisters in $K$ West Basin--Functiona7 Design Criteria, WHC-SD-SNF-FDC-001, Rev. 0, Westinghouse Hanford Company, Richland, Washington.

Prescott, R. D. and D.J. Trimble, 1996, Test Report for Sealed Canister Gas and Liquid Samp7ing Equipment, WHC-SD-SNF-TRP-016, Rev. 0, Westinghouse Hanford Company, Richland, Washington.

Trimble, D. J., 1995a, Data Compilation Report: Gas and Liquid Samples from $K$ West Basin Fuel Storage Canisters, WHC-SD-SNF-DP-001, Rev. O, Westinghouse Hanford Company, Richland, Washington.

Trimble, D. J., 1995b, Data Compilation Report: K West Basin Fuel Storage Canister Liquid Samp7es, WHC-SD-SNF-DP-003, Rev. 0, Westinghouse Hanford Company, Richland, Washington.

Trimble, D. J., 1996a, Data Analysis, K West Basin Canister Liquid and Gas Samples, Gamma Energy Analysis and Mass Spectrometry Data, WHC-SD-SNF-ANAL-007, Rev. 0, Westinghouse Hanford Company, Richland, Washington.

Trimble, D. J., 1996b, Data Analysis of Gas and Liquid From K West Basin Fuel Canisters; 1995 Samp7es, WHC-SD-SNF-ANAL-008, Rev. 0, Westinghouse Hanford Company, Richland, Washington.

Trimble, D. J. and T. L. Welsh, 1997, Cesium-137 in K West Basin Canister Water," HNF-SD-SNF-ANAL-014, Rev. 0, Duke Engineering and Services Hanford, Inc., Richland, Washington.

Trimble, D. J., 1997, Analysis of Water from $K$ West Basin Canisters (Second Campaign), HNF-SD-SNF-TI-048, Rev. 0, Duke Engineering and Services Hanford, Inc., Richland, Washington.

Waymire, G. R., 1996, Specification for Design of the SNF Project, Fue7 Retrieva7 Subproject, WHC-S-0461, Rev. 0, Westinghouse Hanford Company, Rich] and, Washington. 
HNF-SD-SNF-TI-049, Rev. 0

This page intentionally left blank. 
GAS SPECIES ANALYSIS REPORTS FOR $K$ WEST CANISTER SAMPLES

ANALYSES WERE BY PACIFIC NORTHEEST NATIONAL LABORATORY

IN THE HANFORD 325 BUILDING LABORATORY. TYPICAL REPORT SUBMITTAL LETTERS ARE INCLUDED. 
HNF-SD-SNF-TI-049, Rev. O

Page 22

This page intentionally left blank. 
September 10, 1996

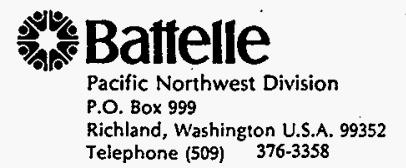

D.J. Trimble

Nuclear Fuel Evaluations

Westinghouse Hanford Co.

PO Box 1970

Richland, WA 99352

Dear Mr. Trimble

Analyses of the K-west basin fuel canister samples received August:29; 1996 and September \$ 5; 1996 are complete. Uncorrected and corrected reports for sample vial background interferences are attached.

Analyses were performed using the PNNL high sensitivity, quantitative, gas mass spectrometer (WC38625). A performance check of the instrument, is made daily, using high purity nitrogen, prior to sample analyses. The Analytical Chemistry Laboratory log-in numbers are 96-06393, 96-06394, 96-06395, 96-06444 and 96-06445. Work package ED6493 was used for this work.

$1 \mathrm{ml}$ aliquots of the samples were removed from the vials using gas sampling syringes. The entire aliquot was injected into a $11.5 \mathrm{cc}$ sample inlet. The pressures and temperature were recorded and the amount of gas in the $1 \mathrm{ml}$ syringe was calculated and reported as the sample size.

If you have any questions concerning this report please free to call 376-3358.

Sincerely yours
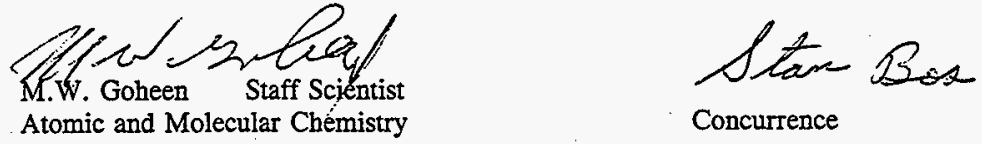

Concurrence 
October 30, 1996

\section{D.J. Trimble}

\section{DESH}

Mail Slot $\mathrm{HO}-40$

Richland, Washington 99352

Dear Mr. Trimble

Analyses of the K-west basin fuel canister samples received October 23; 1996 and October 25, 1996 are complete. Uncorrected and corrected reports for sample vial background interferences are attached.

Analyses were performed using the PNNL high sensitivity, quantitative, gas mass spectrometer (WC38625). A performance check of the instrument, is made daily, using high purity nitrogen, prior to sample analyses. The Analytical Chemistry Laboratory log-in numbers are 97-00284 through 97-00290. Work package ED7032 was used for this work.

$1 \mathrm{ml}$ aliquots of the samples were removed from the vials using gas sampling syringes. The entire aliquot was injected into a $11.5 \mathrm{cc}$ sample inlet. The pressures and temperature were recorded and the amount of gas in the $1 \mathrm{ml}$ syringe was calculated and reported as the sample size.

If you have any questions concerning this report please free to call $376-3358$.

Sincerely yours

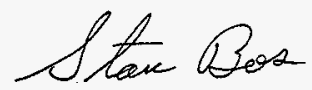

Stanley Bos

Atomic and Molecular Chemistry

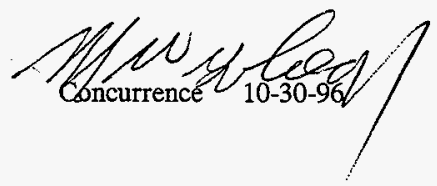

Battelle Boulevard - P.0. Box 999 a Richland, WA 99352 


\section{Pacific Northwest National Laboratory}

From: $\quad 325$ Gas \& Isotopic Mass Spectrometry

Phone: (509) 376-3358/mail slot P7-22

Date: $\quad$ September 16, 1996

Subject: Gas Species Analysis

To:
D.J. Trimble

Analytical procedure: PNL-MA-599 ALO-284

Record book ACL 6

Page 103

Measurement and test equipment WC38625

Sample Id. D014 (Background corrected)

Analysis Date September 12, 1996

Log-in No. $\quad 96-06656$

Sample size $\quad 1.26 \mathrm{cc}$ stp

Liquid found in vial $4.7 \mathrm{ml}$ Mole Estimate of

Percent Precision

\begin{tabular}{|c|c|c|}
\hline Argon & 0.147 & \pm 0.003 \\
\hline Carbon dioxide & 0.091 & \pm 0.002 \\
\hline $\begin{array}{l}\text { Carbon monoxide } \\
\text { Hellum }\end{array}$ & $\begin{array}{r}<0.01 \\
<0.001\end{array}$ & 4 \\
\hline Hydrogen & 86.9 & \pm 0.2 \\
\hline Methane & 0.028 & \pm 0.001 \\
\hline Nitrogen & 103 & \pm 02 \\
\hline oxygen & 2.47 & $\pm 0,05$ \\
\hline Nitrous oxide & $<0.01$ & \pm \\
\hline Other nitrogen oxides & $<0.005$ & \pm \\
\hline Ethane & 00.001 & +1 \\
\hline Other hydrocarbons & $<0,001$ & \pm \\
\hline Krypton 83 & $<0.0005$ & \pm \\
\hline Krypton 84 & $<0.0005$ & \pm \\
\hline Krypton 85 & $<00005$ & \pm \\
\hline Kypton 86 & 0.002 & 10,0005 \\
\hline Xenon 131 & 0.003 & \pm 0.0005 \\
\hline Xenon 132 & 0.004 & \pm 0.0005 \\
\hline Xenon 134 & 0.007 & 200005 \\
\hline Xenon 136 & 0.011 & \pm 0.001 \\
\hline
\end{tabular}

Comments Results corrected by subtracting out gas found in new vials 


\section{Pacific Northwest National Laboratory}

From: $\quad 325$ Gas \& Isotopic Mass Spectrometry

Phone: (509) 376-3358/ mail slot P7-22

Date: $\quad$ September 16, 1996

Subject: Gas Species Analysis

To: $\quad$ D.J. Trimble

Analytical procedure: PNL-MA-599 ALO-284

Record book ACL $6 \quad$ Page 103

Measurement and test equipment WC38625

Sample 1d. D019 (Backgiound corrected)

Analysis Date September 12, 1996

Log-in No. $\quad 96-06657$

Sample size $\quad 1.27 \mathrm{cc}$ stp

Liquid found in vial $3.8 \mathrm{ml}$ Mole Estimate of

Percent Precision

\begin{tabular}{|c|c|c|}
\hline Argon & 0.165 & \pm 0.003 \\
\hline Carbon dioxide & 0.094 & \pm 0.002 \\
\hline Carbon monoxide & $<001$ & 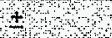 \\
\hline Hellum & 80001 & \pm+1 \\
\hline Hydrogen & 87 & \pm 0.2 \\
\hline Methane & 0.024 & \pm 0.001 \\
\hline Nitrogen & 10.5 & \pm 02 \\
\hline Oxygen & 2,19 & \pm 0.04 \\
\hline Nitrous oxide & $<0.01$ & \pm \\
\hline Other nitrogen oxides & $<0.005$ & \pm \\
\hline Ethane & 80.001 & \pm \\
\hline Other hydrocarbons & 50001 & \pm \\
\hline Krypton 83 & $<0.0005$ & \pm \\
\hline Krypton 84 & $<0.0005$ & \pm \\
\hline Krypton 85 & $<0,0005$ & + \\
\hline Krypton 86 & $<0,0005$ & \pm \\
\hline Xenon 131 & 0.001 & \pm 0.0005 \\
\hline Xenon 132 & 0.002 & \pm 0.0005 \\
\hline Xenon 134 & 0,003 & \pm 0000 \\
\hline Xenon 136 & 0.005 & +00005 \\
\hline
\end{tabular}

Comments Results corrected by subtracting out gas found in new vials 


\section{Pacific Northwest National Laboratory}

From: $\quad 325$ Gas \& Isotopic Mass Spectrometry

Phone: (509) 376-3358/mail slot P7-22

Date: $\quad$ September 6, 1996

Subject: Gas Species Analysis

To: $\quad$ D.J. Trimble

Analytical procedure: PNL-MA-599 ALO-284

Record book ACL $6 \quad$ Page 101

Measurement and test equipment WC38625

Sample Id. D020 (Background corrected)

Analysis Date August 30, 1996

Log-in No. $\quad 96-06395$

Sample size $\quad 1.28 \mathrm{cc}$ stp

Liquid found in vial $1.2 \mathrm{ml} \quad$ Mole Estimate of

Percent Precision

\begin{tabular}{|c|c|c|}
\hline Argon & 0.132 & \pm 0.003 \\
\hline Carbon dioxide & $<0.01$ & \pm \\
\hline Carbon monoxide & $<001$ & 1 \\
\hline Helium & $<0001$ & \pm \\
\hline Hydrogen & 89.2 & \pm 0.2 \\
\hline Methane & 0.018 & \pm 0.001 \\
\hline Nitrogen & +9 & \pm 0.2 \\
\hline Oxygen & 158 & +003 \\
\hline Nitrous oxide & $<0.01$ & \pm \\
\hline Other nitrogen oxides & $<0.005$ & \pm \\
\hline Ethane & $<0001$ & \\
\hline Other hydrocarbons & $<0.001$ & 1 \\
\hline Krypton 83 & $<0.0005$ & \pm \\
\hline Krypton 84 & $<0.0005$ & \pm \\
\hline Krypton 85 & $<0.0005$ & \pm \\
\hline Kypton 86 & 80.0005 & \pm \\
\hline Xenon 131 & 0.001 & \pm 0.0005 \\
\hline Xenon 132 & 0.002 & \pm 0.0005 \\
\hline Xenon 134 & 0003 & \pm 00005 \\
\hline Xenon 136 & 0.005 & \pm 00005 \\
\hline
\end{tabular}

Comments Results corrected by subtracting out gas found in new vials 


\section{Pacific Northwest National Laboratory}

From: $\quad 325$ Gas \& Isotopic Mass Spectrometry

Phone: (509) 376-3358 / mail slot P7-22

Date: $\quad$ September 16, 1996

Subject: Gas Species Analysis

To: $\quad$ D.J. Trimble

Analytical procedure: PNL-MA-599 ALO-284

Record book ACL 6 Page 103

Measurement and test equipment WC38625

Sample Id. D021 (Background corrected)

Analysis Date September 12, 1996

Log-in No. $\quad 96-06658$

Sample size $\quad 1.29 \mathrm{cc}$ stp

Liquid found in vial $1.5 \mathrm{ml}$. Mole Estimate of

Percent Precision

\begin{tabular}{|c|c|}
\hline Argon & \pm 0.004 \\
\hline Carbon dioxide & \pm 0.001 \\
\hline $\begin{array}{l}\text { Carbon monoxide } \\
\text { Helum }\end{array}$ & + + \\
\hline Hydrogen & \pm 0.4 \\
\hline Methane & \pm 0.001 \\
\hline Nitrogen & \pm 0.4 \\
\hline oxygen & +0.07 \\
\hline Nitrous oxide & \pm \\
\hline Other nitrogen oxides & \pm \\
\hline $\begin{array}{l}\text { Ethane } \\
\text { Other hy drocarbons }\end{array}$ & \\
\hline Krypton 83 & \pm \\
\hline Krypton 84 & \pm \\
\hline Krypton 85 & \pm \\
\hline Krypton 86 & \pm \\
\hline Xenon 131 & \pm \\
\hline Xenon 132 & \pm 0.0005 \\
\hline Xenon 134 & +0.0005 \\
\hline Xenon 136 & \pm 0.0005 \\
\hline
\end{tabular}

Comments Results corrected by subtracting out gas found in new vials 


\section{Pacific Northwest National Laboratory}

From: $\quad 325$ Gas \& Isotopic Mass Spectrometry

Phone: (509) 376-3358/mail siot P7-22

Date: September 24, 1996

Subject: Gas Species Analysis

To: $\quad$ D.J. Trimble

Analytical procedure: PNL-MA-599 ALO-284

Record book ACL $6 \quad$ Page 105

Measurement and test equipment WC38625

Sample Id. D026 (Background corrected)

Analysis Date September 20,1996

Log-in No. $\quad 96-06761$

Sample size $\quad 1.26 \mathrm{cc}$ stp

Liquid found in vial $4.5 \mathrm{ml}$ Mole Estimate of

Percent Precision

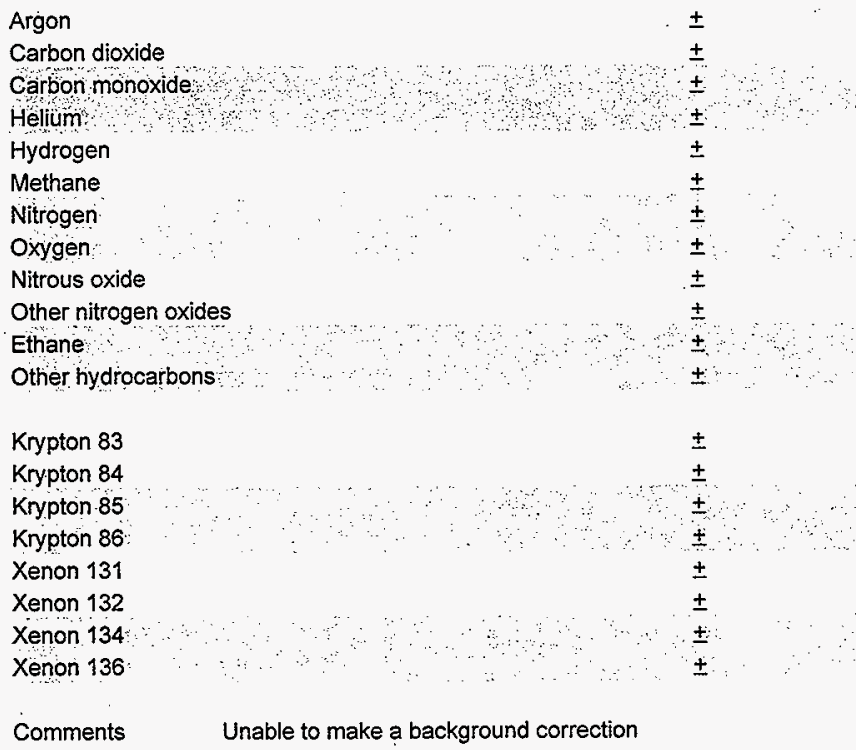




\section{Pacific Northwest National Laboratory}

From: $\quad 325$ Gas \& Isotopic Mass Spectrometry

Phone: (509) 376-3358/ mail slot P7-22

Date: $\quad$ September 10,1996

Subject: Gas Species Analysis

To: $\quad$ D.J. Trimble

Analytical procedure: PNL-MA-599 ALO-284

Record book ACL $6 \quad$ Page 102

Measurement and test equipment WC38625

Sample Id. D027 (Background corrected)

Analysis Date September 06, 1996

Log-in No. 96-06445

Sample size $\quad 1.27 \mathrm{cc}$ stp

Liquid found in vial $1.1 \mathrm{ml}$ Mole Estimate of

Percent Precision

\begin{tabular}{|c|c|c|}
\hline Argon & 0.307 & \pm 0.006 \\
\hline Carbon dioxide & $<0.01$ & \pm \\
\hline $\begin{array}{l}\text { Carbon monoxide } \\
\text { Hellum }\end{array}$ & $<001$ & \pm+4 \\
\hline Hydrogen & 49 & \pm 0.9 \\
\hline Methane & 0.003 & \pm 0.0005 \\
\hline Nitrogen & 492 & \pm 09 \\
\hline Oxygen $\$$ & 146 & +0.03 \\
\hline Nitrous oxide & $<0.01$ & \pm \\
\hline Other nitrogen oxides & $<0.005$ & \pm \\
\hline Ethane & $<0.001$ & \pm \\
\hline Other hyorocarbons & 40001 & + \\
\hline Krypton 83 & $<0.0005$ & \pm \\
\hline Krypton 84 & $<0.0005$ & \pm \\
\hline Krypton 85 & 600005 & \\
\hline Krypton 86 & 0003 & \pm 0.0005 \\
\hline Xenon 131 & 0.003 & \pm 0.0005 \\
\hline Xenon 132 & 0.005 & \pm 0.0005 \\
\hline Xenon 134 & 0008 & \pm 0.0005 \\
\hline Xenon 136 & 0.012 & $\begin{array}{l}+0001 \\
\end{array}$ \\
\hline
\end{tabular}

Comments Results corrected by subtracting out gas found in new vials 


\section{Pacific Northwest National Laboratory}

From: $\quad 325$ Gas \& Isotopic Mass Spectrometry

Phone: $\quad$ (509) 376-3358/ mail slot P7-22

Date: September 16,1996

Subject: Gas Species Analysis

To:

D.J. Trimble

Analytical procedure: PNL-MA-599 ALO-284

Record book ACL $6 \quad$ Page 103

Measurement and test equipment WC38625

Sample ld. D029 (Background corrected)

Analysis Date September 12, 1996

Log-in No. $\quad 96-06659$

Sample size $\quad 1.27 \mathrm{cc}$ stp

Liquid found in vial $4.9 \mathrm{ml}$ Mole Estimate of

Percent Precision

\begin{tabular}{|c|c|c|}
\hline Argon & 0.362 & \pm 0.007 \\
\hline Carbon dioxide & 0.052 & \pm 0.001 \\
\hline Carbon monoxide & $<0.01$ & \\
\hline Hellum & $<0.001$ & \pm+3 \\
\hline Hydrogen & 49.4 & \pm 0.9 \\
\hline Methane & 0.013 & \pm 0.001 \\
\hline Nitrogen & 478 & \pm 0.9 \\
\hline oxygen & 2242 & $\pm 0,05$ \\
\hline Nitrous oxide & $<0.01$ & \pm \\
\hline Other nitrogen oxides & $<0.005$ & \pm \\
\hline Ethane & $<0,001$ & \\
\hline Other hydrocarbons & $<0.001$ & \pm \\
\hline Krypton 83 & $<0.0005$ & \pm \\
\hline Krypton 84 & $<0.0005$ & \pm \\
\hline Krypton 85 & $<00005$ & \pm \\
\hline Krypton 86 & $<0.0005$ & \\
\hline Xenon 131 & 0.001 & \pm 0.0005 \\
\hline Xenon 132 & 0.002 & \pm 0.0005 \\
\hline Xenon 134 & 0.003 & \pm 00005 \\
\hline Xenon 136 & 0,004 & \pm 00005 \\
\hline
\end{tabular}

Comments Results corrected by subtracting out gas found in new vials 


\section{Pacific Northwest National Laboratory}

From: $\quad 325$ Gas \& Isotopic Mass Spectrometry

Phone: . (509) 376-3358/ mail slot P7-22

Date: $\quad$ September 16, 1996

Subject: $\quad$ Gas Species Analysis

To: $\quad$ D.J. Trimble

Analytical procedure: PNL-MA-599 ALO-284

Record book ACL 6 Page 103

Measurement and test equipment WC38625

Sample Id. D030 (Background corrected)

Analysis Date September 12, 1996

Log-in No. $\quad 96-06660$

Sample size $\quad 1.27 \mathrm{cc}$ stp

Liquid found in vial $2.8 \mathrm{ml}$ Mole Estimate of

Percent Precision

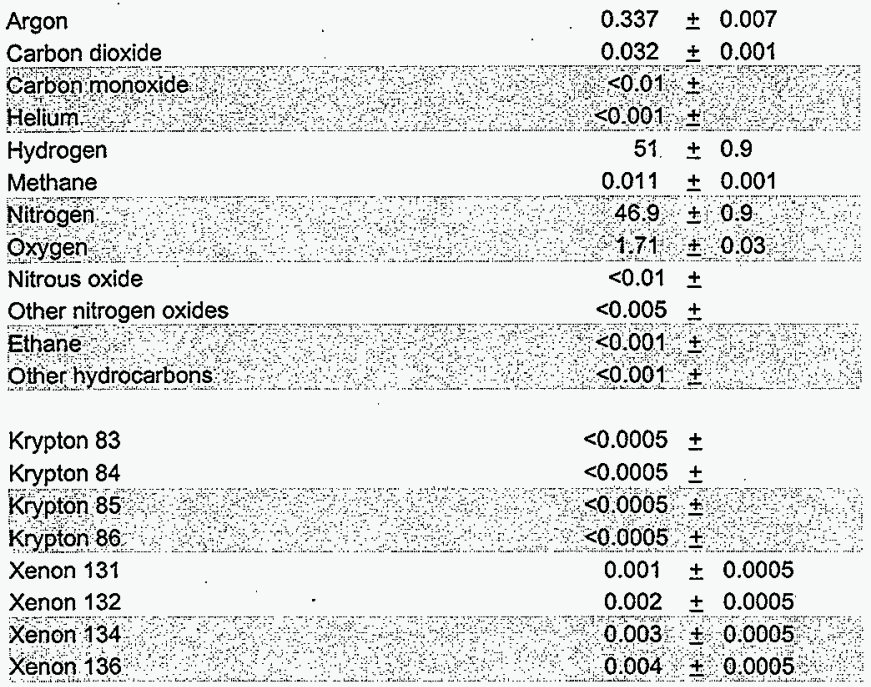

Comments Results corrected by subtracting out gas found in new vials 


\section{Pacific Northwest National Laboratory}

From: $\quad 325$ Gas \& Isotopic Mass Spectrometry

Phone: $\quad$ (509) 376-3358/ mail slot P7-22

Date: $\quad$ September 16, 1996

Subject: Gas Species Analysis

To: $\quad$ D.J. Trimble

Analytical procedure: PNL-MA-599 ALO-284

Record book ACL $6 \quad$ Page 103

Measurement and test equipment WC38625

Sample ld. $\quad$ D034 (Background corrected)

Analysis Date September 12, 1996

Log-in No. $\quad 96-06661$

Sample size $\quad 1.31 \mathrm{cc} \mathrm{stp}$

Liquid found in vial $2.4 \mathrm{ml}$ Mole Estimate of

Percent Precision

\begin{tabular}{|c|c|c|}
\hline Argon & 0.146 & \pm 0.003 \\
\hline Carbon dioxide & 0.041 & \pm 0.001 \\
\hline $\begin{array}{l}\text { Carbon monoxide } \\
\text { Hellum }\end{array}$ & $\begin{array}{l}<0.01 \\
<0,001\end{array}$ & $1+1$ \\
\hline Hydrogen & 60.3 & \pm 0.6 \\
\hline Methane & 0.011 & \pm 0.001 \\
\hline $\begin{array}{l}\text { Nitrogen } \\
\text { Oxygen }\end{array}$ & 104 & $\begin{array}{l}02 \\
+0.6\end{array}$ \\
\hline Nitrous oxide & $<0.01$ & \pm \\
\hline Other nitrogen oxides & $<0.005$ & \pm \\
\hline Ethane & $<0.001$ & \pm \\
\hline Othertydrocarbons & $<0.001$ & \pm \\
\hline Krypton 83 & $<0.0005$ & \pm \\
\hline Krypton 84 & $<0.0005$ & \pm \\
\hline Krypton 85 & $<0.0005$ & \\
\hline Krypton 86 & $<00005$ & \pm \\
\hline Xenon 131 & 0.001 & \pm 0.0005 \\
\hline Xenon 132 & 0.001 & \pm 0.0005 \\
\hline Xenon 134 & 0.002 & \pm 0000 \\
\hline Xenon 136 & 0.003 & \pm 00005 \\
\hline
\end{tabular}

Comments Results corrected by subtracting out gas found in new vials 


\section{Pacific Northwest National Laboratory}

From: $\quad 325$ Gas \& Isotopic Mass Spectrometry

Phone: (509) 376-3358/ mail slot P7-22

Date: September 16, 1996

Subject: Gas Species Analysis

To: D.J. Trimble

Analytical procedure: PNL-MA-599 ALO-284

Record book ACL $6 \quad$ Page 103

Measurement and test equipment WC38625

Sample ld. D034 Dup (Background corrected)

Analysis Date September 12, 1996

Log-in No. $\quad 96-06661$

Sample size $\quad 1.24$ cc stp

Liquid found in vial $2.4 \mathrm{ml}$ Mole Estimate of

Percent Precision

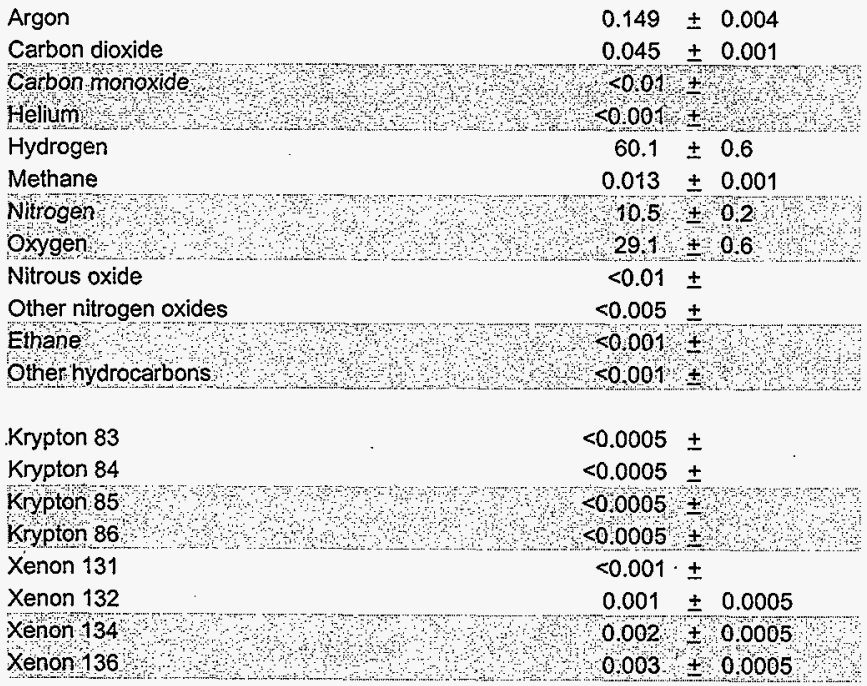

Comments Results corrected by subtracting out gas found in new vials 


\section{Pacific Northwest National Laboratory}

From: $\quad 325$ Gas \& Isotopic Mass Spectrometry

Phone: (509) 376-3358/mail slot P7-22

Date: $\quad$ September 24, 1996

Subject: Gas Species Analysis

To: D.J. Trimble

Analytical procedure: PNL-MA-599 ALO-284

Record book ACL $6 \quad$ Page 104

Measurement and test equipment WC38625

Sample ld. D049 (Background corrected).

Analysis Date September 20,1996

Log-in No. $\quad 96-06758$

Sample size . $\quad 0.77 \mathrm{cc}$ stp

Liquid found in vial $5.4 \mathrm{ml}$ Mole Estimate of

Percent Precision

Argon

Carbon dioxide

Carbon monoxide

Helium

Hydrogen

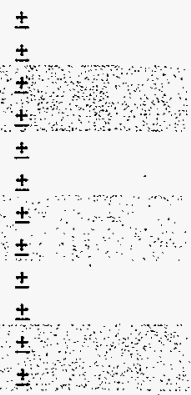

Krypton 83

Krypton 84

Krypton 85

Krypton 86

Xenon 131

Xenon 132

Xenon 134

Xenon 136

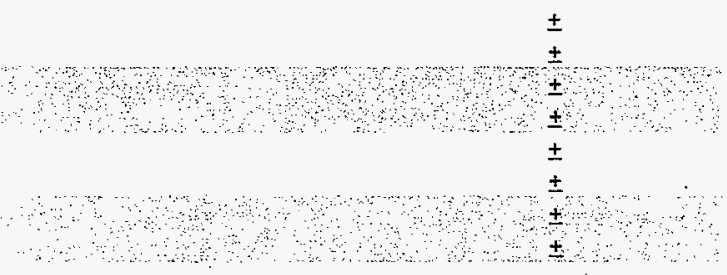

Comments Unable to make a background correction 


\section{Pacific Northwest National Laboratory}

From: $\quad 325$ Gas \& Isotopic Mass Spectrometry

Phone: (509) 376-3358 / mail slot . P7-22

Date: $\quad$ September 24, 1996

Subject: · Gas Species Analysis

To: $\quad$ D.J. Trimble

Analytical procedure: PNL-MA-599 ALO-284

Record book ACL 6 Page $105^{\circ}$

Measurement and test equipment WC38625

Sample ld. D071 (Background corrected)

Analysis Date September 20, 1996

Log-in No. $\quad 96-06759$

Sample size $\quad 1.25 \mathrm{cc}$ stp

Liquid found in vial $1.0 \mathrm{ml}$

$\begin{array}{cl}\text { Mole } & \text { Estimate of } \\ \text { Percent } & \text { Precision }\end{array}$

\begin{tabular}{|c|c|c|}
\hline Argon & 0.081 & \pm 0.002 \\
\hline Carbon dioxide & 0.004 & \pm 0.001 \\
\hline Carbonmonoxide & $<0.01$ & \pm \\
\hline Helium & $<0.001$ & \pm \\
\hline Hydrogen & 92 & \pm 0.1 \\
\hline Methane & 0.024 & \pm 0.001 \\
\hline Nitrogen & 5.1 & \pm 0.1 \\
\hline Oxygen & 2.73 & \pm 0.05 \\
\hline Nitrous oxide & $<0.01$ & \pm \\
\hline Other nitrogen oxides & $<0.005$ & \pm \\
\hline Ethane & $<0001$ & \pm \\
\hline Other hydrocarbon & $<0001$ & \pm \\
\hline Krypton 83 & $<0.0005$ & \pm \\
\hline Krypton 84 & $<0.0005$ & \pm \\
\hline Krypton 85 & $<0.0005$ & \pm \\
\hline Krypton 86 & $<0,0005$ & \pm \\
\hline Xenon 131 & 0.003 & \pm 0.0005 \\
\hline Xenon 132 & 0.004 & \pm 0.0005 \\
\hline Xenon 134 & 0.007 & \pm 0.0005 \\
\hline Xenon 136 & 0.01 & \pm 0.001 \\
\hline
\end{tabular}

Comments Results corrected by subtracting out gas found in new vials 
From: $\quad 325$ Gas \& 1sotopic Mass Spectrometry

Phone: $\quad$ (509) 376-3358 / mail slot P7-22

Date: October 01, 1996

Subject: Gas Species Analysis

To:

D.J. Trimble

Analytical procedure: PNL-MA-599 ALO-284

Record book ACL $6 \quad$ Page 106

Measurement and test equipment WC38625

Sample ld. D072 (Backgiround corrected)

Analysis Date September 26, 1996

Log-in No. $\quad 96-06838$

Sample size $\quad 0.24 \mathrm{cc}$ stp

Liquid found in vial $1.2 \mathrm{ml}$

Room temperature $\quad 69.6^{\circ} \mathrm{F} \quad$ Mole Estimate of

Percent Precision.

\begin{tabular}{|c|c|c|}
\hline Argon & 0.36 & \pm 0.007 \\
\hline Carbon dioxide & 0.038 & \pm 0.001 \\
\hline Carbon monoxide & $<0,1$ & \pm \\
\hline Heliumm & $<0.001$ & \pm \\
\hline Hydrogen & 66 & \pm 0.5 \\
\hline Methane & 0.104 & \pm 0.002 \\
\hline Nitrogen & 266 & \pm 0.5 \\
\hline Oxygen & 6.9 & \pm 0.1 \\
\hline Nitrous oxide & $<0.01$ & \pm \\
\hline Other nitrogen oxides & $<0.005$ & \pm \\
\hline Etharie & $<0.001$ & \pm \\
\hline Other hydrocarbons & $<0001$ & \pm \\
\hline Krypton 83 & $<0.0005$ & \pm \\
\hline Krypton 84 & $<0.0005$ & \pm \\
\hline Krypton 85 & $<0.0005$ & \pm \\
\hline Krypton 86 & $<0,0005$ & \pm \\
\hline Xenon 131 & $<0.0005$ & \pm \\
\hline Xenon 132 & 0.002 & \pm 0.0005 \\
\hline Xenon 134 & 0.004 & \pm 0.0005 \\
\hline Xenon 136 & 0.007 & \pm 0.0005 \\
\hline
\end{tabular}

Comments 


\section{Pacific Northwest National Laboratory}

From: $\quad 325$ Gas \& Isotopic Mass Spectrometry

Phone: $\quad$ (509) 376-3358/mail slot P7-22

Date: $\quad$ September 24, 1996

Subject: Gas Species Analysis

To: $\quad$ D.J. Trimble

Analytical procedure: PNL-MA-599 ALO-284

Record book ACL 6

Page 105

Measurement and test equipment WC38625

Sample Id. D074 (Background corrected)

Analysis Date September 20,1996

Log-in No. $\quad 96-06760$

Sample size $\quad 0.55 \mathrm{cc}$ stp

Liquid found in vial $0.4 \mathrm{ml}$

Mole Estimate of

Percent Precision
Argon

Carbon dioxide

Carbon monoxide

Helium

Hydrogen

Methane

Nitrogen

Oxygen

Nitrous oxide

Other nitrogen oxides

Ethane

Other hydrocarbons

Krypton 83

Krypton 84

Krypton 85

Kryptón 86

Xenon 131

Xenon 132

Xenon 134

Xenon 136

Comments
$0.87 \pm 0.02$

$<0.001 \pm$

$<0.01 \pm$ $<0.001 \pm$

$7.6 \pm 0.2$

$0.01 \pm 0.001$

$718 \pm 0.4$

$19.8 \pm 0.4$

$<0.001 \pm$

$<0.005 \pm$

$<0.001 \pm$

$<0.001 \pm$

$<0.0005 \pm$ $<0.0005 \pm$ $<0.0005 \pm$

$<0.0005 \pm$

$<0.0005 \pm$

$<0.0005 \pm$

$<0.0005 \pm$

$<0.0005 \pm$

Results corrected by subtracting out gas found in new vials 


\section{Pacific Northwest National Laboratory}

From: $\quad 325$ Gas \& Isotopic Mass Spectrometry

Phone: (509) 376-3358/mail slot P7-22

Date: $\quad$ October 01, 1996

Subject: Gas Species Analysis

To: $\quad$ D.J. Trimble

Analytical procedure: PNL-MA-599 ALO-284

Record book ACL 6

Page 106

Measurement and test equipment WC38625

Sample Id.

D097 (Background corrected)

Analysis Date

September 26, 1996

Log-in No.

96-06839

Sample size $\quad 1.29$ cc stp

Liquid found in vial $0.5 \mathrm{ml}$

Room temperature $\quad 68.9^{\circ} \mathrm{F}$

Mole Estimate of

Percent Precision

\begin{tabular}{|c|c|c|}
\hline Argon & 0.07 & \pm 0.001 \\
\hline Carbon dioxide & $<0.001$ & \pm \\
\hline Carbon monoxide & $<0.01$ & \pm \\
\hline Heliump & $<0.001$ & \pm \\
\hline Hydrogen & 94 & \pm 0.1 \\
\hline Methane & 0.022 & \pm 0.001 \\
\hline itrogen & 469 & \pm 0.09 \\
\hline Oxygen & 1.15 & \pm 0.02 \\
\hline Nitrous oxide & $<0.001$ & \pm \\
\hline Other nitrogen oxides & $<0.005$ & \pm \\
\hline Ethane & $<0.001$ & \pm \\
\hline ther hydrocarbons & $<0001$ & \\
\hline Krypton 83 & $<0.0005$ & \pm \\
\hline Krypton 84 & $<0.0005$ & \pm \\
\hline Krypton 85 & $<0,0005$ & \pm \\
\hline Krypton 86 & $<0.0005$ & \pm \\
\hline Xenon 131 & 0.003 & \pm 0.0005 \\
\hline Xenon 132 & 0.005 & \pm 0.0005 \\
\hline Xenon 134 & 0.009 & \pm 0.001 \\
\hline Xenon 136 & 0.013 & \pm 0001 \\
\hline
\end{tabular}

Comments 


\section{Pacific Northwest National Laboratory}

From: $\quad 325$ Gas \& Isotopic Mass Spectrometry

Phone: $\quad$ (509) 376-3358/mail slot P7-22

Date: $\quad$ October 01, 1996

Subject: . Gas Species Analysis

To: $\quad$ D.J. Trimble

Analytical procedure: PNL-MA-599 ALO-284

Record book ACL $6 \quad$ Page 106

Measurement and test equipment WC38625

Sample ld. D101 (Background corrected)

Analysis Date . September 26, 1996

Log-in No. $\quad 96-06840$

Sample size $\quad 1.25 \mathrm{cc}$ stp

Liquid found in vial $3.5 \mathrm{ml}$

Room temperature $68.5^{\circ} \mathrm{F} \quad$ Mole Estimate of

Percent Precision

\begin{tabular}{|c|c|c|}
\hline Argon & 0.148 & \pm 0.003 \\
\hline Carbon dioxide & $<0.001$ & \pm \\
\hline Carbonmonoxide & $<0.01$ & \pm \\
\hline Hélium & $<0.001$ & \pm \\
\hline Hydrogen & 89.5 & \pm 0.2 \\
\hline Methane & 0.025 & \pm 0.001 \\
\hline Nitrogen & 88 & \pm 0.2 \\
\hline oxygen & 1.53 & \pm 0.03 \\
\hline Nitrous oxide & $<0.001$ & \pm \\
\hline Other nitrogen oxides & $<0.005$ & \pm \\
\hline Ethane & $<0001$ & \pm \\
\hline ther hydrocarbons & $<0.001$ & \pm \\
\hline Krypton 83 & $<0.0005$ & \pm \\
\hline Krypton 84 & $<0.0005$ & \pm \\
\hline Krypton 85 & $<0.0005$ & \pm \\
\hline Krypton 86 & $<0.0005$ & \pm \\
\hline Xenon 131 & 0.003 & \pm 0.0005 \\
\hline Xenon 132 & 0.005 & \pm 0.0005 \\
\hline Xenon 134 & 0.008 & \pm 0.0005 \\
\hline Xenon 136 & 0.012 & \pm 0.001 \\
\hline
\end{tabular}

Comments 


\section{Pacific Northwest National Laboratory}

From: $\quad 325$ Gas \& Isotopic Mass Spectrometry

Phone: (509) 376-3358/ mail slot P7-22

Date: October 01, 1996

Subject: Gas Species Analysis

To: $\quad$ D.J. Trimble

Analytical procedure: PNL-MA-599 ALO-284

Record book ACL $6 \quad$ Page 106

Measurement and test equipment WC38625

Sample 1d. D102 (Backǵround corrected)

Analysis Date September 26, 1996

Log-in No. $\quad 96-06841$

Sample size $\quad 1.29$ cc stp

Liquid found in vial $0.1 \mathrm{ml}$

Room temperature $\quad 68.2^{\circ} \mathrm{F} \quad$ Mole Estimate of

Percent Precision

\begin{tabular}{|c|c|c|}
\hline Argon & 0.07 & \pm 0.001 \\
\hline Carbon dioxide & $<0.001$ & \pm \\
\hline Carbonmonoxide & $<001$ & \pm \\
\hline Heliúm & $<0001$ & \pm \\
\hline Hydrogen & 93.8 & \pm 0.2 \\
\hline Methane & 0.014 & \pm 0.001 \\
\hline Nitrogen & 5.1 & \pm 0.1 \\
\hline Oxygen & 0.94 & \pm 0.02 \\
\hline Nitrous oxide & $<0.001$ & \pm \\
\hline Other nitrogen oxides & $<0.005$ & \pm \\
\hline Ethane & $<0,001$ & \pm \\
\hline Other hydrocarbons & $<0001$ & $\pm \quad$ \\
\hline Krypton 83 & $<0.0005$ & \pm \\
\hline Krypton 84 & $<0.0005$ & \pm \\
\hline Krypton 85 & $<0.0005$ & \pm \\
\hline Krypton 86 & 0.002 & \pm 0.0005 \\
\hline Xenon 131 & 0.004 & \pm 0.0005 \\
\hline Xenon 132 & 0.008 & \pm 0.0005 \\
\hline Xenon 134 & 0.013 & $\pm 0,001$ \\
\hline Xenon 136 & 0.02 & \pm 0.001 \\
\hline
\end{tabular}

Comments 


\section{Pacific Northwest National Laboratory}

From: $\quad 325$ Gas \& Isotopic Mass Spectrometry

Phone: (509) 376-3358 / mall slot P7-22

Date: October 01, 1996

Subject: Gas Species Analysis

To:

D.J. Trimble

Analytical procedure: PNL-MA-599 ALO-284

Record book ACL $6 \quad$ Page 106

Measurement and test equipment WC38625

Sample ld. D103 (Background corrected)

Analysis Date September 26, 1996

Log-in No. $\quad 96-06842$

Sample size $\quad 1.27$ cc stp

Liquid found in vial $0.5 \mathrm{ml}$

Room temperature $68.2^{\circ} \mathrm{F} \quad$ Mole Estimate of

Percent Precision

\begin{tabular}{|c|c|c|}
\hline Argon & 0.065 & \pm 0.001 \\
\hline Carbon dioxide & $<0.001$ & \pm \\
\hline Carbon monoxide & $<0.01$ & \% \\
\hline Helium & $<0.001$ & \pm \\
\hline Hydrogen & 94 & \pm 0.2 \\
\hline Methane & 0.037 & \pm 0.001 \\
\hline Nitrogen & 4.63 & \pm 0.09 \\
\hline Oxygen & 1.17 & \pm 0.02 \\
\hline Nitrous oxide & $<0.001$ & \pm \\
\hline Other nitrogen oxides & $<0.005$ & \pm \\
\hline Ethane & $<0.001$ & \pm \\
\hline Other hydrocarbons & $<0.001$ & $\pm \quad$ \\
\hline Krypton 83 & $<0.0005$ & \pm \\
\hline Krypton 84 & 0.002 & \pm 0.0005 \\
\hline Krypton 85 & $<00005$ & $\pm \quad-$ \\
\hline Krypton 86 & 0.005 & \pm 0.0005 \\
\hline Xenon 131 & 0.008 & \pm 0.0005 \\
\hline Xenon 132 & 0.015 & \pm 0.001 \\
\hline Xenor 134 & $0: 024$ & \pm 0.001 \\
\hline Xenon 136 & 0.036 & \pm 0.001 \\
\hline
\end{tabular}

Comments 


\section{Pacific Northwest National Laboratory}

From: $\quad 325$ Gas \& Isotopic Mass Spectrometry

Phone: (509) 376-3358/ mail slot P7-22

Date: $\quad$ October 01, 1996

Subject: Gas Species Analysis

To: $\quad$ D.J. Trimble

Analytical procedure: PNL-MA-599 ALO-284

Recoro book ACL $6 \quad$ Page 106

Measurement and test equipment WC38625

Sample ld. D104 (Background corrected)

Analysis Date September 27, 1996

Log-in No. $\quad 96-06843$

Sample size $\quad 0.15 \mathrm{cc} \mathrm{stp}$

Liquid found in vial $0.3 \mathrm{ml}$

Room temperature $69.8^{\circ} \mathrm{F} \quad$ Mole Estimate of

Percent Precision

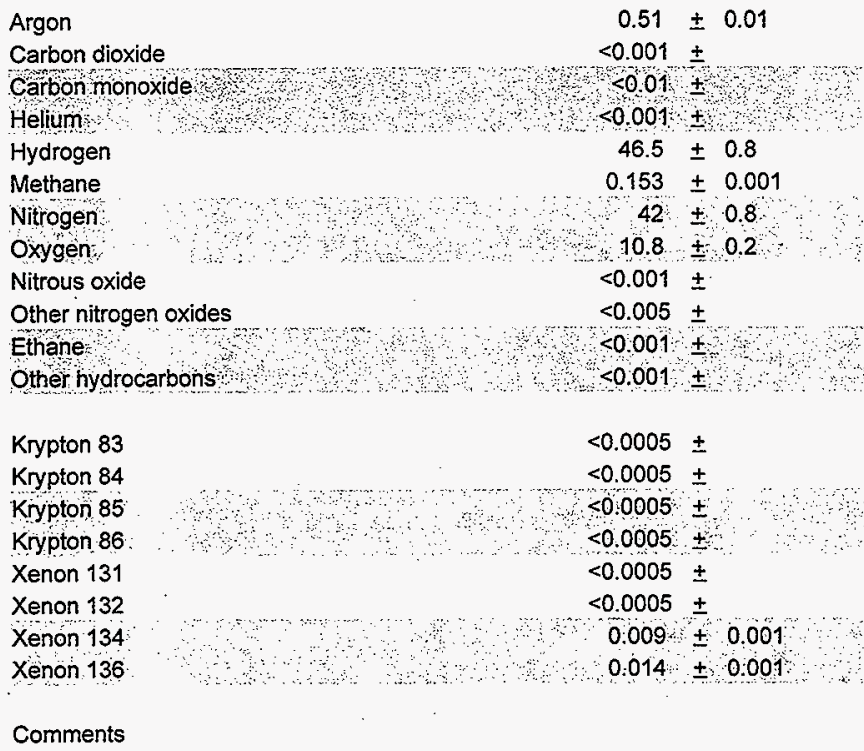




\section{Pacific Northwest National Laboratory}

From: $\quad 325$ Gas \& Isotopic Mass Spectrometry

Phone: - (509) 376-3358 / mail slot P7-22

Date: $\quad$ October 29, 1996

Subject: Gas Species Analysis

To: DJ Trimble

Analytical procedure: PNL-MA-599 ALO-284

Record book ACL 6 Page 111

Measurement and test equipment WC38625

Sample ld.

D165 (Backgiround Corrected)

Analysis Date October 23, 1996

Log-in No.

Sample size $\quad 1.28 \mathrm{cc}$ stp

Liquid found in vial

Room temperature $\quad 69.8^{\circ} \mathrm{F}$

Mole Estimate of

Percent Precision

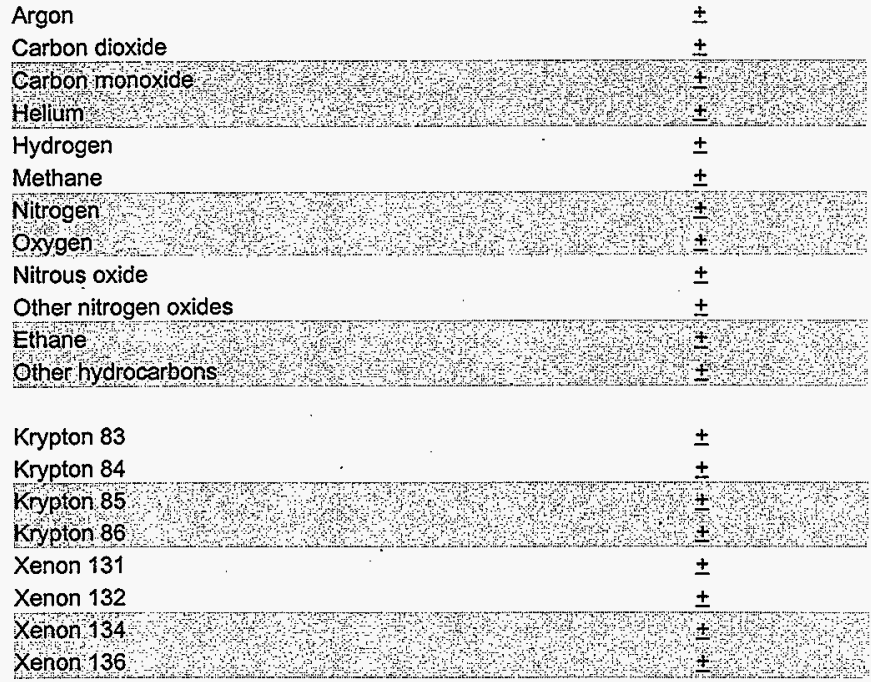

Comments Unable to make a background correction 


\section{Pacific Northwest National Laboratory}

From: $\quad 325$ Gas \& Isotopic Mass Spectrometry

Phone: $\quad$ (509) 376-3358/ mail slot P7-22

Date: $\quad$ October 29, 1996

Subject: Gas Species Analysis

To: $\quad$ DJ Trimble

Analytical procedure: PNL-MA-599 ALO-284

Record book ACL $6 \quad$ Page 111

Measurement and test equipment WC38625

Sample Id.

D165 Dup (Background Corrected)

Analysis Date October 23, 1996

Log-in No.

Sample size $\quad 1.20 \mathrm{cc}$ stp

Liquid found in vial

Room temperature $\quad 69.1^{\circ} \mathrm{F}$

$\begin{array}{cl}\text { Mole } & \text { Estimate of } \\ \text { Percent } & \text { Precision }\end{array}$
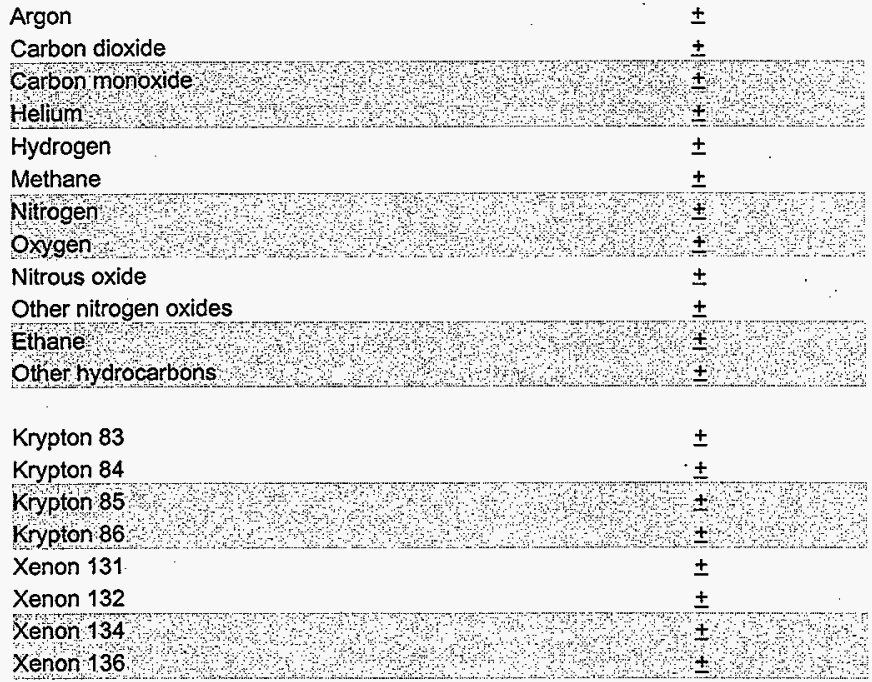

Comments Unable to make a background correction 


\section{Pacific Northwest National Laboratory}

From: $\quad 325$ Gas \& Isotopic Mass Spectrometry

Phone: $\quad$ (509) 376-3358 / mail slot P7-22

Date: $\quad$ October 29, 1996

Subject: Gas Species Analysis

To: DJ Trimble

Analytical procedure: PNL-MA-599 ALO-284

Record book $\mathrm{ACL} 6 \quad$ Page 111

Measurement and test equipment WC38625

Sample Id. D166 (Background Corrected)

Analysis Date October 23, 1996

Log-in No. $\quad 97.00285$

Sample size $\quad 1.25 \mathrm{cc} \mathrm{stp}$

Liquid found in vial

Room temperature $\quad 69.6^{\circ} \mathrm{F}$ Mole Estimate of

Percent Precision

\begin{tabular}{|c|c|c|}
\hline Argon & 0.164 & \pm 0.004 \\
\hline Carbon dioxide & 0.032 & $\pm 0: 004$ \\
\hline Carbon monoxide & $<0,01$ & $+4+$ \\
\hline Hellum & $<0001$ & $1+1$ \\
\hline Hydrogen & 84.1 & \pm 0.2 \\
\hline Methane & 0.017 & \pm 0.002 \\
\hline Nifrogen & 123 & \pm 02 \\
\hline Oxygen & 328 & \pm 0.07 \\
\hline Nitrous oxide & $<0.01$ & \pm \\
\hline Other nitrogen oxides & $<0.005$ & \pm \\
\hline Ethane & $<0,01$ & \pm \\
\hline Other hydrocarbons & $<0.01$ & \pm+1 \\
\hline Krypton 83 & $<0.0005$ & \pm \\
\hline Krypton 84 & 0.002 & \pm 0.0005 \\
\hline Kupton 85 & $\$ 0,0005$ & \pm \\
\hline Krypton 86 & 0.003 & $\pm 0,0005$ \\
\hline Xenon 131 & 0.005 & \pm 0.001 \\
\hline Xenon 132 & 0.008 & \pm 0.001 \\
\hline Xenon 134 & 0013 & 40.002 \\
\hline Xenon 136 & 0019 & \pm 0.002 \\
\hline
\end{tabular}

Comments 


\section{Pacific Northwest National Laboratory}

From: $\quad 325$ Gas \& Isotopic Mass Spectrometry

Phone: $\quad$ (509) 376-3358 / mail slot P7-22

Date: October 29, 1996

Subject: Gas Species Analysis

To: $\quad$ DJ Trimble

Analytical procedure: PNL-MA-599 ALO-284

Record book ACL 6 Page 111

Measurement and test equipment WC38625

Sample Id. D175 (Backgiround Corrected)

Analysis Date October 28, 1996

Log-in No. $\quad 97-00286$

Sample size $\quad 1.28$

Liquid found in vial

Room temperature $\quad 70.7^{\circ} \mathrm{F} \quad$ Mole Estimate of

Percent Precision

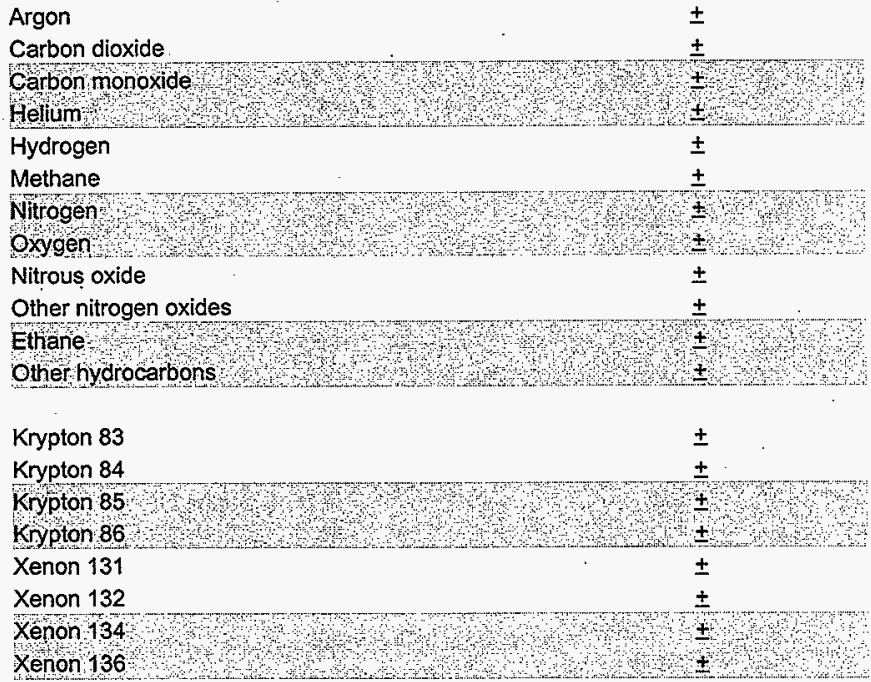

Comments Unable to make a background correction 


\section{Pacific Northwest National Laboratory}

From: $\quad 325$ Gas \& Isotopic Mass Spectrometry

Phone: $\quad$ (509) 376-3358 / mail slot P7-22

Date: $\quad$ October 29, 1996

Subject: Gas Species Analysis

To: $\quad$ DJ Trimble

Analytical procedure: 'PNL-MA-599 ALO-284

Record book ACL 6 Page 111

Measurement and test equipment WC38625

Sample id. D176 (Backgiround Corrected)

Analysis Date Ocfober 28, 1996

Log-in No. $\quad 97-00287$

Sample size $\quad 0.86$

Liquid found in vial

Room temperature $\quad 70.2^{\circ} \mathrm{F} \quad$ Mole Estimate of

Percent Precision

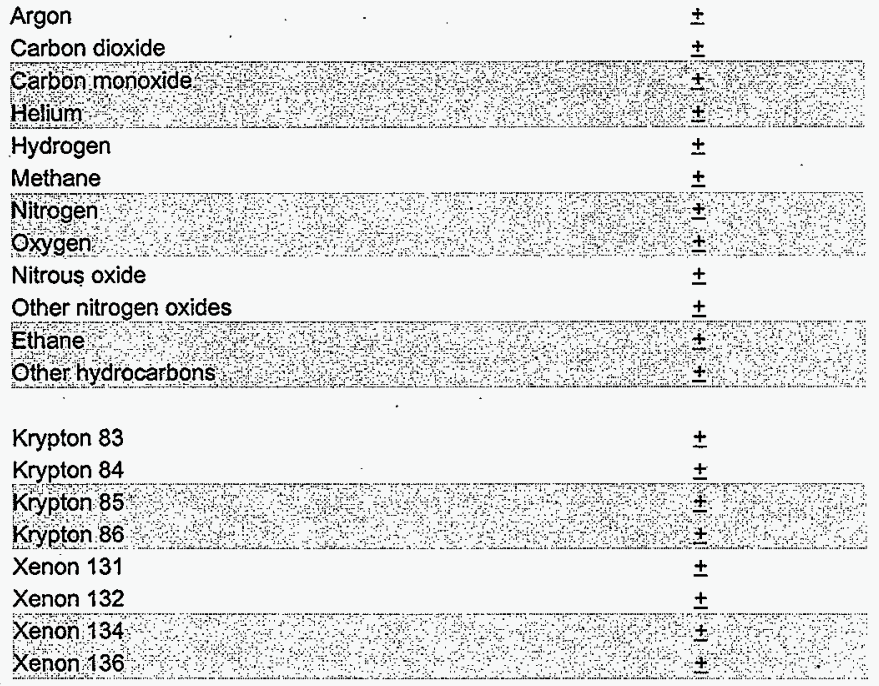

Comments Unable to make a background correction 


\section{Pacific Northwest National Laboratory}

From: $\quad 325$ Gas \& 1sotopic Mass Spectrometry

Phone: $\quad$ (509) 376-3358 / mail slot P7-22

Date: $\quad$ October 29, 1996

Subject: Gas Species Analysis

To:

DJ Trimble

Analytical procedure: PNL-MA-599 ALO-284

Record book ACL 6 Page 111

Measurement and test equipment WC38625

Sample Id. D178 (Backgiround Corrected)

Analysis Date . October 23,1996

Log-in No. $\quad 97-00288$

Sample size $\quad 1.27 \mathrm{cc} \mathrm{stp}$

Liquid found in vial

Room temperature $\quad 69.1^{\circ} \mathrm{F}$ Mole Estimate of

Percent Precision

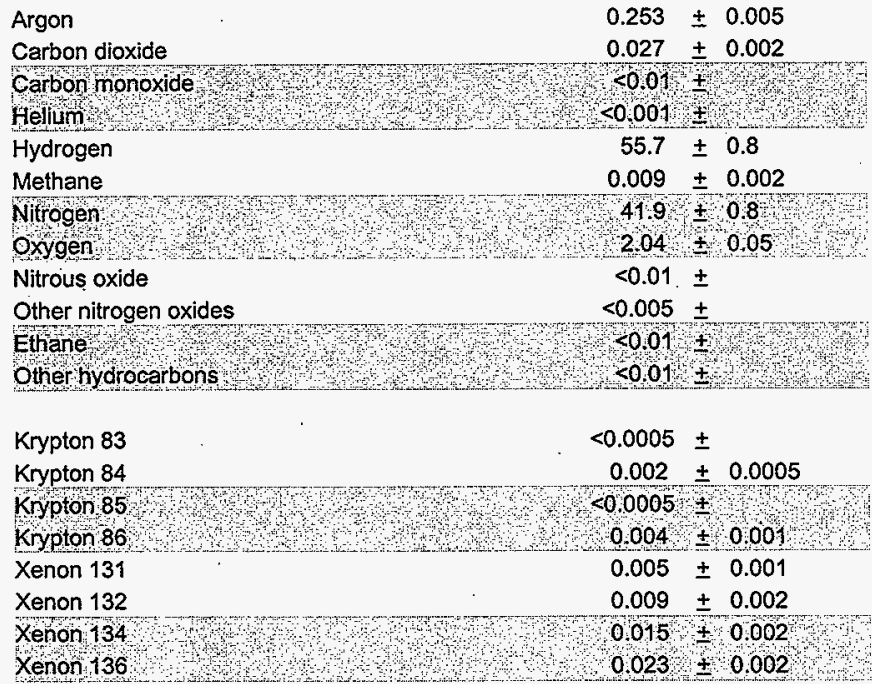

Comments 


\section{Pacific Northwest National Laboratory}

From: $\quad 325$ Gas \& Isotopic Mass Spectrometry

Phone: (509) 376-3358/mail slot P7-22

Date: $\quad$ October 29, 1996

Subject: Gas Species Analysis

To: $\quad$ DJ Trimble

Analytical procedure: PNL-MA-599 ALO-284

Record book ACL $6 \quad$ Page 111

Measurement and test equipment WC38625.

Sample Id. D181 (Background Corrected)

Analysis Date October 23, 1996

Log-in No. $\quad 97-00289$

Sample size $\quad 1.44 \mathrm{ccstp}$

Liquid found in vial

Room temperature $76.8^{\circ} \mathrm{F}$. Mole Estimate of

Percent Precision

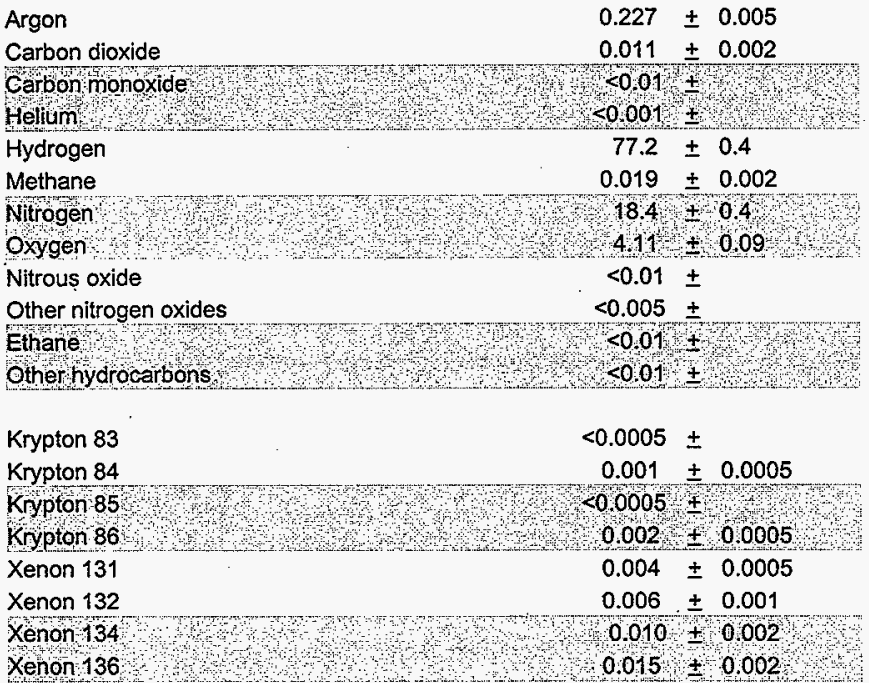

Comments 


\section{Pacific Northwest National Laboratory}

From: $\quad 325$ Gas \& Isotopic Mass Spectrometry

Phone: $\quad$ (509) 376-3358/ mail slot P7-22

Date: $\quad$ October 29, 1996

Subject: Gas Species Analysis

To: DJ Trimble

Analytical procedure: PNL-MA-599 ALO-284

Record book ACL 6 Page 111

Measurement and test equipment WC38625

Sample ld. D182 (Backǵround Corrected)

Analysis Date October 23, 1996

Log-in No. $\quad 97-00290$

Sample size $\quad 1.26 \mathrm{cc}$ stp

Liquid found in vial

Room temperature $\quad 77.9^{\circ} \mathrm{F} \quad$ Mole Estimate of

Percent Precision

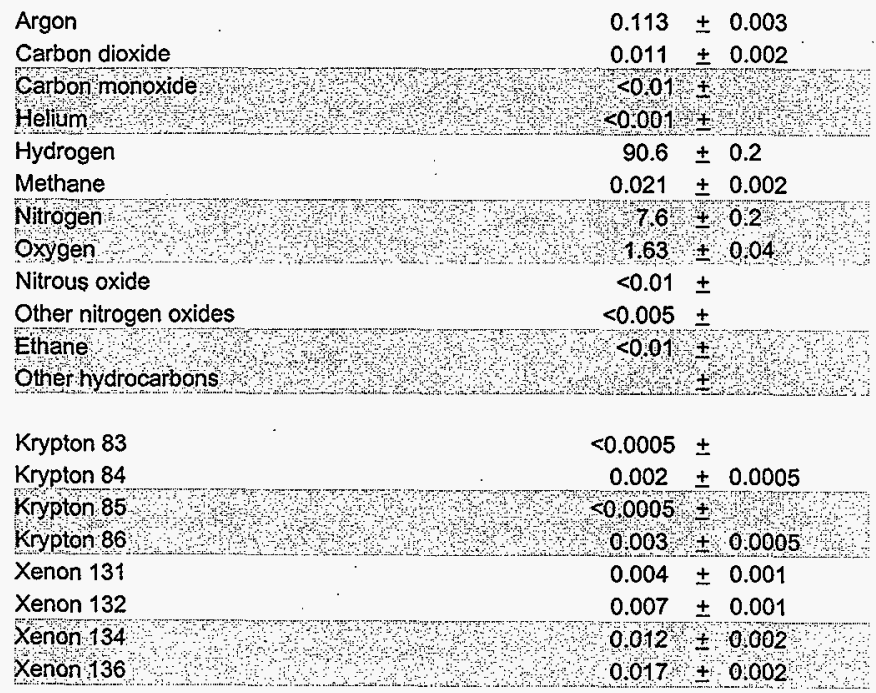

Comments 


\section{Pacific Northwest National Laboratory}

From: $\quad 325$ Gas \& isotopic Mass Spectrometry

Phone: (509) 376-3358/ mail slot P7-22

Date: $\quad$ September 6,1996

Subject: Gas Species Analysis

To: $\quad$ D.J. Trimble

Analytical procedure: PNL-MA-599 ALO-284

Record book ACL $6 \quad$ Page 101

Measurement and test equipment WC38625

Sample Id. E01 (Background corrected)

Analysis Date August 30,1996

Log-in No. $\quad 96-06393$

Sample size $\quad 1.30 \mathrm{cc}$ stp

Liquid found in vial $1.8 \mathrm{ml}$ Mole Estimate of

Percent Precision

\begin{tabular}{|c|c|c|}
\hline Argon & 0.145 & \pm 0.003 \\
\hline Carbon dioxide & 0.105 & \pm 0.002 \\
\hline $\begin{array}{l}\text { Carbon monoxide } \\
\text { Hellum }\end{array}$ & $\begin{array}{l}<0,01 \\
<0001\end{array}$ & \pm+ \\
\hline Hydrogen & 89.8 & \pm 0.2 \\
\hline Methane & 0.018 & \pm 0.001 \\
\hline Nitrogen & 8.6 & +0.2 \\
\hline oxygen & 134 & +0.03 \\
\hline Nitrous oxide & $<0.01$ & \pm \\
\hline Other nitrogen oxides & $<0.005$ & \pm \\
\hline Ethane & $<0.001$ & \pm \\
\hline Other hydrocarbons & $<0.001$ & \pm \\
\hline Krypton 83 & $<0.0005$ & \pm \\
\hline Krypton 84 & $<0.0005$ & \pm \\
\hline Kopton 85 & 80.0005 & + \\
\hline Krypton 86 & 0001 & \pm 0.0005 \\
\hline Xenon 131 & 0.002 & \pm 0.0005 \\
\hline Xenon 132 & 0.002 & \pm 0.0005 \\
\hline Xenon 134 & 0.004 & \pm 00005 \\
\hline Xenon 136 & 0006 & \pm 0.0005 \\
\hline
\end{tabular}

Comments Results corrected by subtracting out gas found in new vials 


\section{Pacific Northwest National Laboratory}

From: $\quad 325$ Gas \& Isotopic Mass Spectrometry

Phone: (509) 376-3358/mail slot P7-22

Date: $\quad$ September 6, 1996

Subject: Gas Species Analysis

To: $\quad$ D.J. Trimble

Analytical procedure: PNL-MA-599 ALO-284

Record book ACL 6 Page 101

Measurement and fest equipment WC38625

Sample ld. $\quad$ E01 (Dup) Bàckground corrected

Analysis Date . September 3, 1996

Log-in No. $\quad 96-06393$

Sample size $\quad 0.99 \mathrm{cc}$ stp

Liquid found in viai $1.8 \mathrm{ml}$. Mole Estimate of

Percent Precision

\begin{tabular}{|c|c|c|}
\hline Argon & 0.153 & \pm 0.003 \\
\hline Carbon dioxide & 0.134 & \pm 0.003 \\
\hline Carbonmonoxide & 18001 & +4 \\
\hline Heliam & $<0001$ & \pm+4 \\
\hline Hydrogen & 88.8 & \pm 0.2 \\
\hline Methane & 0.018 & \pm 0.001 \\
\hline Nifrogen & 92 & \pm 0.2 \\
\hline Oxygen & 1.65 & \pm 0.03 \\
\hline Nitrous oxide & $<0.01$ & \pm \\
\hline Other nitrogen oxides & $<0.005$ & \pm \\
\hline Ethane & $<0001$ & \pm \\
\hline other fyodrocarbons & $-0,001$ & \pm \\
\hline Krypton 83 & $<0.0005$ & \pm \\
\hline Kypton 84 & $<0.0005$ & \pm \\
\hline Krypton 85 & $<0,0005$ & \\
\hline Kypton 86 & 0.001 & \pm 00005 \\
\hline Xenon 131 & 0.001 & \pm 0.0005 \\
\hline Xenon 132 & 0.002 & \pm 0.0005 \\
\hline Xenon 134 & 0,004 & $\pm 0,0005$ \\
\hline Xenon 136 & 0.005 & \pm 0.0005 \\
\hline
\end{tabular}

Comments Results corrected by subtracting out gas found in new vials 


\section{Pacific Northwest National Laboratory}

From: $\quad 325$ Gas \& Isotopic Mass Spectrometry

Phone: (509) 376-3358/ mail slot P7-22

Date: September 6,1996

Subject: Gas Species Analysis

To: D.J. Trimble

Analytical procedure: PNL-MA-599 ALO-284

Record book ACL 6 Page 101

Measurement and test equipment WC38625

Sample ld. E02 (Background corrected)

Analysis Date August 30,1996

Log-in No. $\quad 96-06394$

Sample size $\quad 0.21 \mathrm{cc}$ stp

Liquid found in vial $0.05 \mathrm{ml}$

$\begin{array}{cl}\text { Mole } & \text { Estimate of } \\ \text { Percent } & \text { Precision }\end{array}$

\begin{tabular}{|c|c|c|}
\hline Argon & 0.132 & \pm 0.003 \\
\hline Carbon dioxide & $<0.01$ & \pm \\
\hline Carboh monoxide & $<0,0$ & + \\
\hline Helium & $<0001$ & $\pm N 1$ \\
\hline Hydrogen & 89.7 & \pm 0.2 \\
\hline Methane & 0.019 & \pm 0.001 \\
\hline Nitrogen & 177 & \pm 01 \\
\hline oxygen $\mathrm{HU}$ & 3.14 & \pm 006 \\
\hline Nitrous oxide & $<0.01$ & \pm \\
\hline Other nitrogen oxides & $<0.005$ & \pm \\
\hline Efhane & $<0,001$ & \pm \\
\hline other hydrocarbons & $<0,001$ & \pm+ \\
\hline Krypton 83 & $<0.0005$ & \pm \\
\hline Krypton 84 & 0.003 & \pm 0.0005 \\
\hline Krypton 85 & $<00005$ & \pm \\
\hline Krypton 86 & 0003 & \pm 00005 \\
\hline Xenon 131 & 0.003 & \pm 0.0005 \\
\hline Xenon 132 & 0.004 & \pm 0.0005 \\
\hline Xenon 134 & 0.007 & +0.0005 \\
\hline Xenon 136 & 0009 & $+0,0005$ \\
\hline
\end{tabular}

Comments Results corrected by subtracting out gas found in new vials 


\section{Pacific Northwest National Laboratory}

From: $\quad 325$ Gas \& Isotopic Mass Spectrometry

Phone: $\quad$ (509) 376-3358 / mail slot P7-22

Date: $\quad$ September 10,1996

Subject: Gas Species Analysis

To: $\quad$ D.J. Trimble

Anaiytical procedure: PNL-MA-599 ALO-284

Record book ACL $6 \quad$ Page 102

Measurement and test equipment WC38625

Sample ld. E06 (B́ackground corrected)

Analysis Date September 06,1996

Log-in No. $\quad 96-06444$

Sample size $\quad 1.22 \mathrm{cc}$ stp

Liquid found in vial $3.5 \mathrm{ml}$ Mole Estimate of

Percent Precision

\begin{tabular}{|c|c|c|}
\hline Argon & 0.169 & \pm 0.003 \\
\hline Carbon dioxide & 0.043 & \pm 0.001 \\
\hline $\begin{array}{l}\text { Carbon monoxide, } \\
\text { Hellum }\end{array}$ & $\begin{array}{r}80.01 \\
<0.001\end{array}$ & \pm \\
\hline Hydrogen & 84.3 & \pm 0.2 \\
\hline Methane & 0.016 & \pm 0.001 \\
\hline Nitrogen & 12 & $\pm 02 \%$ \\
\hline oxygen & 3.44 & \pm 0.07 \\
\hline Nitrous oxide & $<0.01$ & \pm \\
\hline Other nitrogen oxides & $<0.005$ & \pm \\
\hline Ethane & $<0.001$ & \pm \\
\hline Other hydrocarbons & $<0,001$ & \pm \\
\hline Krypton 83 & $<0.0005$ & \pm \\
\hline Krypton 84 & $<0.0005$ & \pm \\
\hline Krypton 85 & $<0,0005$ & \\
\hline Krypton 86 & 0003 & \pm 0.0005 \\
\hline Xenon 131 & 0.004 & \pm 0.0005 \\
\hline Xenon 132 & 0.007 & \pm 0.0005 \\
\hline Xenon 134 & 0,011 & \pm 0.001 \\
\hline Xeron 136 & 0.016 & \pm 0.001 \\
\hline
\end{tabular}

Comments Results corrected by subtracting out gas found in new vials 


\section{Pacific Northwest National Laboratory}

From: $\quad 325$ Gas \& Isotopic Mass Spectrometry

Phone: (509) 376-3358/ mail slot P7-22

Date: $\quad$ September 10, 1996

Subject: Gas Species Analysis

To: $\quad$ D.J. Trimble

Analytical procedure: PNL-MA-599 ALO-284

Record book ACL $6 \quad$ Page 102

Measurement and test equipment. WC38625

Sample ld. E06 (Dup) : Background corrected

Analysis Date September 06, 1996

Log-in No. $\quad 96-06444$

Sample size $\quad 1.13 \mathrm{cc}$ stp

Liquid found in vial $3.5 \mathrm{ml}$

$\begin{array}{cl}\text { Mole } & \text { Estimate of } \\ \text { Percent } & \text { Precision }\end{array}$

\begin{tabular}{|c|c|c|}
\hline Argon & 0.165 & \pm 0.003 \\
\hline Garbon dioxide & 0.041 & \pm 0.001 \\
\hline $\begin{array}{l}\text { Carbon monoxide } \\
\text { Hellum }\end{array}$ & $\begin{array}{l}<001 \\
<0.001\end{array}$ & +4 \\
\hline Hydrogen & 84.6 & \pm 0.2 \\
\hline Methane & 0.016 & \pm 0.001 \\
\hline Nitrogen & 117 & \pm 02 \\
\hline Oxygen & 3.45 & \pm 0.07 \\
\hline Nitrous oxide & $<0.01$ & \pm \\
\hline Other nitrogen oxides & $<0.005$ & \pm \\
\hline Ethane & $<0.001$ & \pm \\
\hline Other hy drocarbons & $<0.001$ & \pm \\
\hline & & + \\
\hline Krypton 84 & $<0.0005$ & $\begin{array}{l}+ \\
\pm\end{array}$ \\
\hline Krypton 85 & $<0,0005$ & \pm \\
\hline Kryton 86 & 0003 & +00005 \\
\hline Xenon 131 & 0.004 & \pm 0.0005 \\
\hline Xenon 132 & 0.007 & \pm 0.0005 \\
\hline Xenon 134 & 0.011 & \pm 0.0005 \\
\hline Xenon 136 & 0016 & +00005 \\
\hline
\end{tabular}

Comments Results corrected by subtracting out gas found in new vials 


\section{Pacific Northwest National Laboratory}

From: $\quad 325$ Gas \& Isotopic Mass Spectrometry

Phone: (509) 376-3358/ mail slot P7-22

Date: October 01, 1996

Subject: Gas Species Analysis

To: $\quad$ D.J. Trimble

Analytical procedure: PNL-MA-599 ALO-284

Record book ACL $6 \quad$ Page 106

Measurement and test equipment WC38625

Sample Id. E09 (Background corrected)

Analysis Date September 27, 1996

Log-in No. $\quad 96-06844$

Sample size $\quad 1.25 \mathrm{cc}$ stp

Liquid found in vial $1.9 \mathrm{m!}$

Room temperature $\quad 70.7^{\circ} \mathrm{F}$ Mole Estimate of

Percent Precision

\begin{tabular}{|c|c|c|}
\hline Argon & 0.07 & \pm 0.001 \\
\hline Carbon dioxide & 0.007 & \pm 0.001 \\
\hline Carbon monoxide & $<0.01$ & \pm \\
\hline Helium & $<0.001$ & $\pm \quad$ \\
\hline Hydrogen & 95.2 & \pm 0.2 \\
\hline Methane & 0.02 & \pm 0.001 \\
\hline Nitrogen & 3.8 & \pm 0.08 \\
\hline Oxygen & 0.9 & \pm 0.02 \\
\hline Nitrous oxide & $<0.01$ & \pm \\
\hline Other nitrogen oxides & $<0.005$ & \pm \\
\hline Ethane & $<0.001$ & \pm \\
\hline Other hydrocarbons & $<0.001$ & $\pm \quad \square$ \\
\hline Krypton 83 & $<0.0005$ & \pm \\
\hline Krypton 84 & 0.002 & \pm 0.0005 \\
\hline Krypton 85 & $<00005$ & \pm \\
\hline Krypton 86 & 0.003 & \pm 0.0005 \\
\hline Xenon 131 & 0.005 & \pm 0.0005 \\
\hline Xenon 132 & 0.009 & \pm 0.001 \\
\hline Xenon 134 & 0.014 & \pm 0.001 \\
\hline Xenon 136 & 0.021 & \pm 0.001 \\
\hline
\end{tabular}

Comments 


\section{Pacific Northwest National Laboratory}

From: $\quad 325$ Gas \& Isotopic Mass Spectrometry

Phone: $\quad$ (509) $376-3358 /$ mail slot P7-22

Date: $\quad$ October 01, 1996

Subject: Gas Species Analysis

To: $\quad$ D.J. Trimble

Analytical procedure: PNL-MA-599 ALO-284

Record book ACL $6 \quad$ Page 106

Measurement and test equipment WC38625

Sample ld. E09 Dup (Background corrected)

Analysis Date September 27, 1996

Log-in No. $\quad 96-06844$

Sample size $\quad 1.16 \mathrm{cc}$ stp

Liquid found in vial $1.9 \mathrm{ml}$

Room temperature $68.5^{\circ} \mathrm{F} \quad$ Mole Estimate of

Percent Precision

Argon

$0.072 \pm 0.001$

Carbon dioxide

$0.009 \pm 0.001$

Carbon monoxide

$<0.01 \pm$

Helium :

Hydrogen

$<0.001+$

Methane

$95 \pm 0.2$

Nitrogen

$0.018 \pm 0.001$

oxygen

$3.9 \pm 0.08$

Nitrous oxide

$0.95 \pm 0.02$

Other nitrogen oxides

$<0.01 \pm$

Ethane

Other hydrocarbons

$<0.005 \pm$

$<0.001$

$<0.001$

$\pm$

Krypton 83

$<0.0005 \pm$

Krypton 84

$<0.0005 \pm$

Krypton 85

$<0.0005 \pm$

Krypton 86

$0.003+0.0005$

Xenon 131

$0.005 \pm 0.0005$

Xenon 132

$0.009 \pm 0.001$

Xenon 134

$0.014 \pm 0001$

Xenon 136

$0.021 \pm 0.001$

Comments 


\section{Pacific Northwest National Laboratory}

From: $\quad 325$ Gas \& isotopic Mass Spectrometry

Phone: $\quad$ (509) 376-3358 / mail slot P7-22

Date: $\quad$ October 01, 1996

Subject: Gas Species Analysis

To: $\quad$ D.J. Trimble

Analytical procedure: PNL-MA-599 ALO-284

Record book ACL $6 \quad$ Page 106

Measurement and test equipment WC38625

Sample ld. E11 (Background corrected)

Analysis Date September 27, 1996

Log-in No. $\quad 96-06845$

Sample size $\quad 1.26 \mathrm{cc}$ stp

Liquid found in vial $0 \mathrm{ml}$

Room temperature $\quad 70.7^{\circ} \mathrm{F} \quad$ Mole Estimate of

Percent Precision

\begin{tabular}{|c|c|c|}
\hline Argon & 0.047 & \pm 0.001 \\
\hline Carbon dioxide & $<0.001$ & \pm \\
\hline arbon monoxide & $<0.1$ & + \\
\hline Helium & $<0,001$ & \pm \\
\hline Hydrogen & 97.2 & \pm 0.2 \\
\hline Methane & 0.016 & \pm 0.001 \\
\hline Nitrogen & 2.17 & \pm 0.04 \\
\hline Oxygen & 0.49 & \pm 0.01 \\
\hline Nitrous oxide & $<0.001$ & \pm \\
\hline Other nitrogen oxides & $<0.005$ & \pm \\
\hline thane & $<0.009$ & \pm \\
\hline ther hyd & $<0,001$ & \pm \\
\hline Krypton 83 & $<0.0005$ & \pm \\
\hline Krypton 84 & 0.002 & \pm 0.0005 \\
\hline Krypton 85 & $<0: 0005$ & \pm \\
\hline Krypton 86 & 0.004 & \pm 00005 \\
\hline Xenon 131 & 0.005 & \pm 0.0005 \\
\hline Xenon 132 & 0.009 & \pm 0.001 \\
\hline Xenon 134 & $0: 015$ & \pm 0001 \\
\hline Xenon 136 & 0,022 & \pm 0.001 \\
\hline
\end{tabular}

Comments 


\section{Pacific Northwest National Laboratory}

From: $\quad 325$ Gas \& Isotopic Mass Spectrometry

Phone: $\quad$ (509) 376-3358/ mail slot P7-22

Date: $\quad$ October 01, 1996

Subject: Gas Species Analysis

To: D.J. Trimble

Analytical procedure: PNL-MA-599 ALO-284

Record book ACL $6 \quad$ Page 106

Measurement and test equipment WC38625

Sample ld. E12 (Background corrected)

Analysis Date September 27, 1996

Log-in No. $\quad 96-06846$

Sample size $\quad 1.21 \mathrm{cc}$ stp

Liquid found in vial $0 \mathrm{ml}$

Room temperature $\quad 69.3^{\circ} \mathrm{F}$

$\begin{array}{ll}\text { Estimate of } \\ \text { Percent } & \text { Precision }\end{array}$

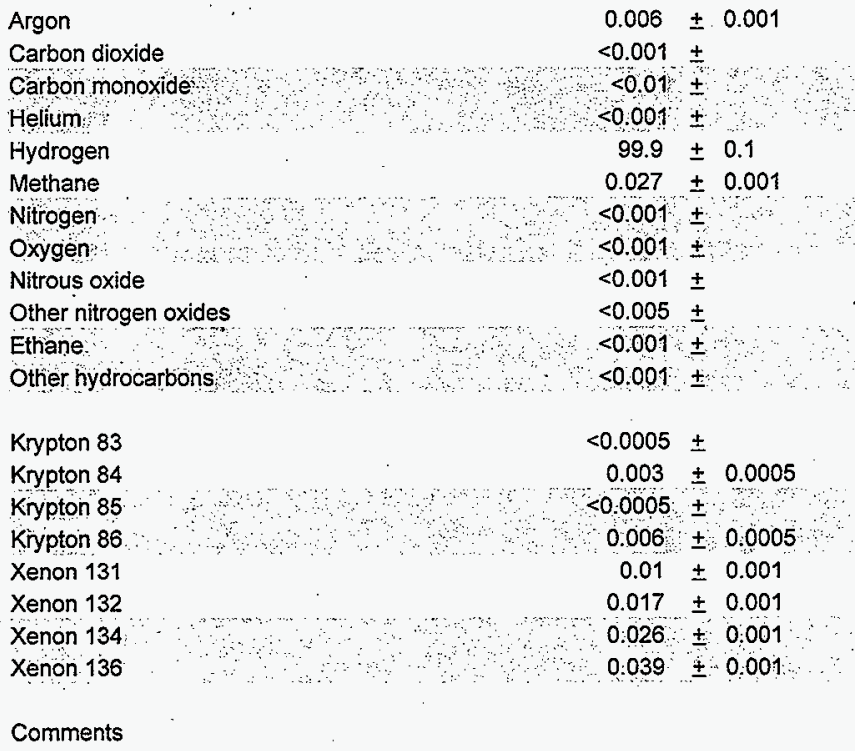




\section{Pacific Northwest National Laboratory}

From: $\quad 325$ Gas \& Isotopic Mass Spectrometry

Phone: (509) 376-3358/ mail slot P7-22

Date: $\quad$ September 16, 1996

Subject: Gas Species Analysis

To: $\quad$ D.J. Trimble

Analytical procedure: PNL-MA-599 ALO-284

Record book ACL $6 \quad$ Page 103

Measurement and test equipment WC38625

Sample Id. E13 (Background corrected)

Analysis Date September 12, 1996

Log-in No. $\quad 96-06655$

Sample size. $\quad 1.16 \mathrm{cc}$ stp

Liquid found in vial $0.5 \mathrm{ml}$ Mole Estimate of

Percent Precision

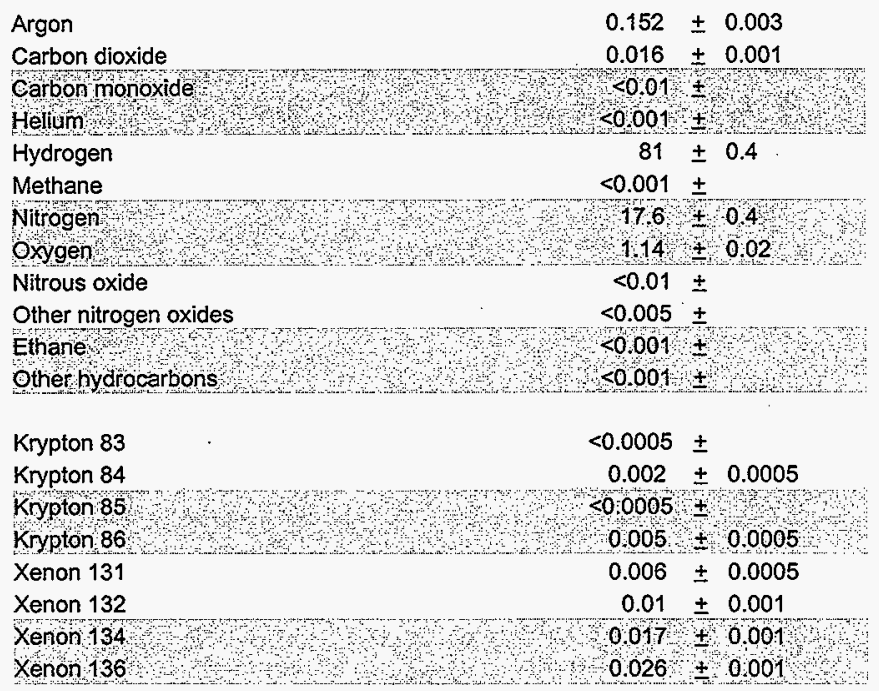

Comments Results corrected by subtracting out gas found in new vials 


\section{Pacific Northwest National Laboratory}

From: $\quad 325$ Gas \& isotopic Mass Spectrometry

Phone: $\quad$ (509) 376-3358/mail slot P7-22

Date: $\quad$ September 24, 1996

Subject: Gas Species Analysis

To: D.J. Trimble

Analytical procedure: PNL-MA-599 ALO-284

Record book ACL $6 \quad$ Page 104

Measurement and test equipment WC38625

Sample Id. E-14 (Background corrected)

Analysis Date September 20,1996

Log-in No. $\quad 96-06753$

Sample size $\quad 1.22 \mathrm{cc}$ stp

Liquid found in vial $1.5 \mathrm{ml}$

$\begin{array}{cl}\text { Mole } & \text { Estimate of } \\ \text { Percent } & \text { Precision }\end{array}$

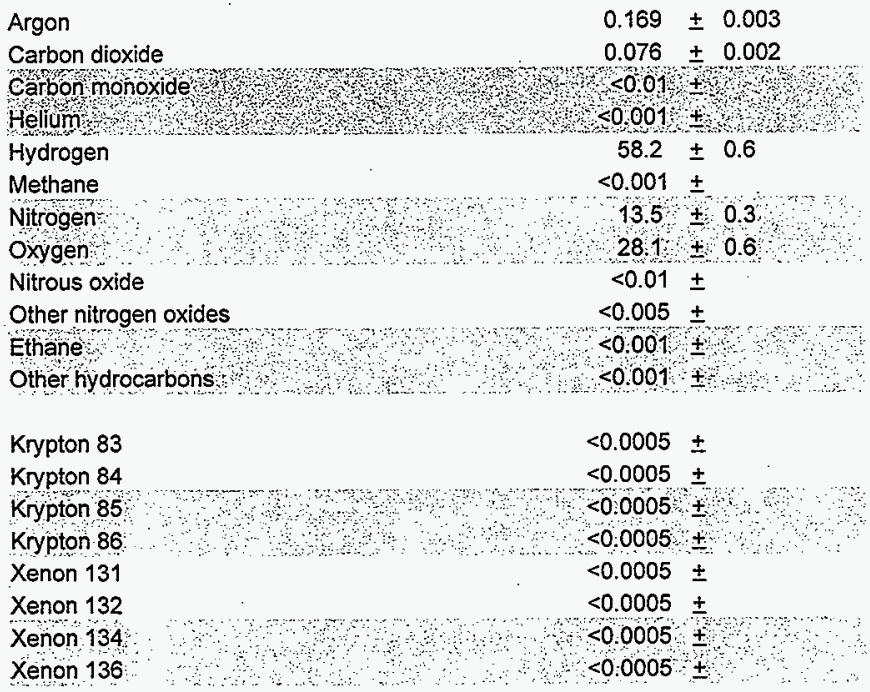

Comments Results corrected by subtracting out gas found in new vials 


\section{Pacific Northwest National Laboratory}

From: $\quad 325$ Gas \& Isofopic Mass Spectrometry

Phone: (509) 376-3358/mail slot P7-22

Date: $\quad$ October 01, 1996

Subject: Gas Species Analysis

To: $\quad$ D.J. Trimble

Analytical procedure: PNL-MA-589 ALO-284

Record book ACL 6 . Page 106

Measurement and test equipment WC38625

Sample Id. E15 (Background corrected)

Analysis Date September 27, 1996

Log-in No. $\quad 96-06847$

Sample size $\quad 0.18 \mathrm{cc}$ stp

Liquid found in vial $1.1 \mathrm{ml}$

Room temperature $\quad 68.0^{\circ} \mathrm{F} \quad$ Mole Estimate of

Percent Precision

\begin{tabular}{|c|c|c|}
\hline Argon & 0.48 & \pm 0.01 \\
\hline Carbon dioxide & 1.08 & \pm 0.02 \\
\hline Carbon monox & $<0.01$ & \pm \\
\hline Helium & $<0.001$ & \pm \\
\hline Hydrogen & 52.8 & \pm 0.9 \\
\hline Methane & 0.077 & \pm 0.002 \\
\hline Nitrogen & 26.4 & \pm 0.5 \\
\hline Oxygen & 191 & \pm 04 \\
\hline Nitrous oxide & $<0.01$ & \pm \\
\hline Other nitrogen oxides & $<0.005$ & \pm \\
\hline Ethane & $<0.001$ & \pm \\
\hline Other hydrocarbons & $<0.001$ & $\pm \quad$ \\
\hline Krypton 83 & $<0.0005$ & \pm \\
\hline Krypton 84 & $<0.0005$ & \pm \\
\hline Kypton 85 & $<0.0005$ & \pm \\
\hline Krypton 86 & $<00005$ & \pm \\
\hline Xenon 131 & $<0.0005$ & \pm \\
\hline Xenon 132 & $<0.0005$ & \pm \\
\hline Xenon 134 & $<0.0005$ & \pm \\
\hline Xenon 136 & 0.004 & \pm 0.0005 \\
\hline
\end{tabular}




\section{Pacific Northwest National Laboratory}

From: $\quad 325$ Gas \& Isotopic Mass Spectrometry

Phone: $\quad$ (509) 376-3358/ mail slot P7-22

Date: $\quad$ September 24, 1996

Subject: Gas Species Analysis

To: D.J. Trimble

Analytical procedure: PNL-MA-599 ALO-284

Record book ACL $6 \quad$ Page 104

Measurement and test equipment WC38625

Sample Id. E-16 (Background corrected)

Analysis Date September 20,1996

Log-in No. $\quad 96-06754$

Sample size $\quad 1.21 \mathrm{cc}$ stp

Liquid found in vial $1 \mathrm{ml}$ Mole Estimate of

Percent Precision

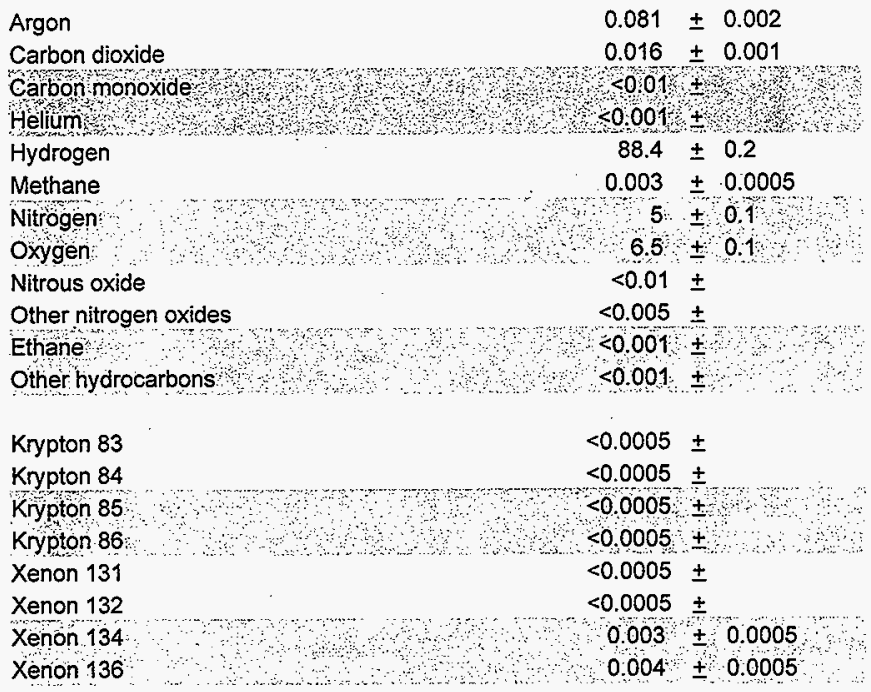

Comments Results corrected by subtracting out gas found in new vials 


\section{Pacific Northwest National Laboratory}

From: $\quad 325$ Gas \& Isotopic Mass Spectrometry

Phone: (509) 376-3358/ mail slot P7-22

Date: $\quad$ October 08, 1996

Subject: Gas Species Analysis

To: $\quad$ D.J. Trimble

Analytical procedure: PNL-MA-599 ALO-284

Record book ACL $6 \quad$ Page 107

Measurement and test equipment WC38625

Sample ld. E-18 (Background corrected)

Analysis Date October 4, 1996

Log-in No. $\quad 97-00029$

Sample size $\quad 1.28 \mathrm{cc} \mathrm{stp}$

Liquid found in vial $0.05 \mathrm{ml}$

Room temperature $\quad 72.0^{\circ} \mathrm{F} \quad$ Mole Estimate of

Percent Precision

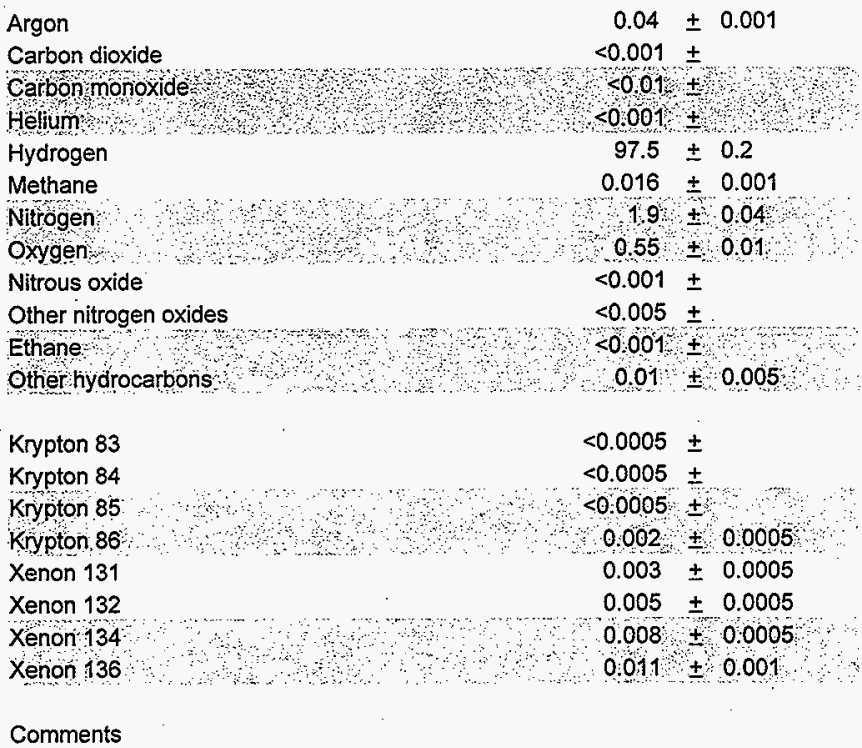




\section{Pacific Northwest National Laboratory}

From: $\quad 325$ Gas \& Isotopic Mass Spectrometry

Phone: $\quad$ (509) 376-3358/ mail slot P7-22

Date: $\quad$ October 08, 1996

Subject: Gas Species Analysis

To: $\quad$ D.J. Trimble

Analytical procedure: PNL-MA-599 ALO-284

Record book ACL 6 Page 107

Measurement and test equipment WC386625

Sample Id. E-18 Dup (Bàckground corrected)

Analysis Date October 4, 1996

Log-in No. $\quad 97-00029$

Sample size $\quad 1.19 \mathrm{cc}$ stp

Liquid found in vial $0.05 \mathrm{ml}$

Room temperature $68.7^{\circ} \mathrm{F} \quad$ Mole Estimate of

Percent Precision

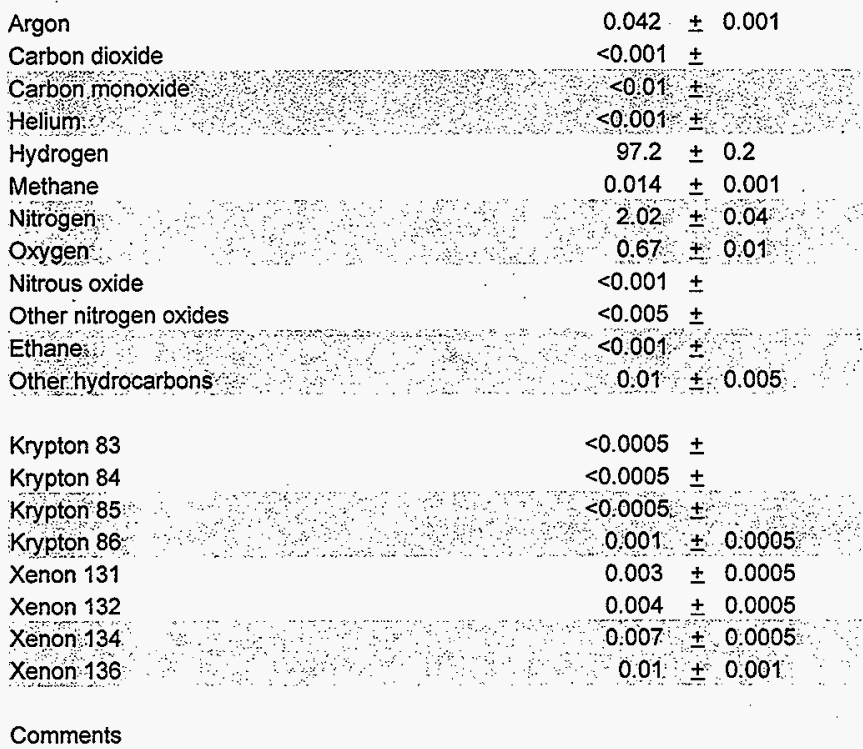




\section{Pacific Northwest National Laboratory}

From: $\quad 325$ Gas \& Isotopic Mass Spectrometry

Phone: $\quad$ (509) 376-3358 / mail slot P7-22

Date: $\quad$ September 24, 1996

Subject: Gas Species Analysis

To: $\quad$ D.J. Trimble

Analytical procedure: PNL-MA-599 ALO-284

Record book ACL $6 \quad$ Page 104

Measurement and test equipment WC38625

Sample ld. E-20 (Background corrected)

Analysis Date September 20,1996

Log-in No. $\quad 96-06755$

Sample size $\quad 1.24 \mathrm{cc}$ stp

Liquid found in vial $0.1 \mathrm{ml}$ Mole Estimate of

Percent Precision

\begin{tabular}{|c|c|c|}
\hline Argon & 0.042 & \pm 0.001 \\
\hline Carbon dioxide & $<0.001$ & \pm \\
\hline Carbon monoxide & $<0.01$ & + \\
\hline Helium & $<0.001$ & \pm \\
\hline Hydrogen & 96.4 & \pm 0.1 \\
\hline Methane & 0.002 & \pm 0.0005 \\
\hline Nitrogen & 178 & \pm 0.04 \\
\hline Oxygen & 174 & \pm 003 \\
\hline Nitrous oxide & $<0.001$ & \pm \\
\hline Other nitrogen oxides & $<0.005$ & \pm \\
\hline Ethane & $<0.001$ & \pm \\
\hline ther hydrocar & $<0001$ & \pm \\
\hline Krypton 83 & $<0.0005$ & \pm \\
\hline Krypton 84 & $<0.0005$ & \pm \\
\hline Krypton 85 & $<0.0005$ & \pm \\
\hline Krypton 86 & $<0.0005$ & \pm \\
\hline Xenon 131 & $<0.0005$ & \pm \\
\hline Xenon 132 & $<0.0005$ & \pm \\
\hline Xenon 134 & 0.002 & +00005 \\
\hline Xenon 136 & 0.002 & \pm 00005 \\
\hline
\end{tabular}

Comments Results corrected by subtracting out gas found in new vials 


\section{Pacific Northwest National Laboratory}

From: $\quad 325$ Gas \& Isotopic Mass Spectrometry

Phone: $\quad$ (509) 376-3358 / mail slot P7-22

Date: September 24, 1996

Subject: Gas Species Analysis

To: $\quad$ D.J. Trimble

Analytical procedure: PNL-MA-599 ALO-284

Record book ACL $6 \quad$ Page 104

Measurement and test equipment WC38625

Sample Id. E-22 (Background corrected)

Analysis Date September 20,1996

Log-in No. $\quad 96-06756$

Sample size $\quad 1.22 \mathrm{cc}$ stp

Liquid found in vial $0.6 \mathrm{ml}$

$\begin{array}{ll}\text { Mole } & \text { Estimate of } \\ \text { Percent } & \text { Precision }\end{array}$

\begin{tabular}{|c|c|c|}
\hline Argon & 0.069 & \pm 0.001 \\
\hline Carbon dioxide & 0.006 & \pm 0.001 \\
\hline Carbon monoxide & $<0.01$ & \pm \\
\hline Helium & $<0.001$ & \pm \\
\hline Hydrogen & 74.4 & \pm 0.4 \\
\hline Methane & 0.008 & \pm 0.001 \\
\hline Nitrogen & 4.04 & \pm 0.08 \\
\hline Oxygen & 214 & \pm 0.4 \\
\hline Nitrous oxide & $<0.01$ & \pm \\
\hline Other nitrogen oxides & $<0.005$ & \pm \\
\hline Ethane & $<0.001$ & \pm \\
\hline Other hydrocarbons & -0.001 & \pm \\
\hline Krypton 83 & $<0.0005$ & \pm \\
\hline Krypton 84 & $<0.0005$ & \pm \\
\hline Krypton 85 & $<0.0005$ & \pm \\
\hline Krypton 86 & $<0.0005$ & \pm \\
\hline Xenon 131 & $<0.0005$ & \pm \\
\hline Xenon 132 & 0.001 & \pm 0.0005 \\
\hline Xenon 134 & 0.002 & \pm 0.0005 \\
\hline Xenon 136 & 0.003 & \pm 0.0005 \\
\hline
\end{tabular}

Comments Results corrected by subtracting out gas found in new vials 


\section{Pacific Northwest National Laboratory}

From: $\quad 325$ Gas \& Isotopic Mass Spectrometry

Phone: (509) 376-3358 / mail slot P7-22

Date: $\quad$ October 08, 1996

Subject: Gas Species Analysis

To: D.J. Trimble

Analytical procedure: PNL-MA-599 ALO-284

Record book ACL $6 \quad$ Page 107

Measurement and test equipment 'WC38625

Sample Id. E-25 (Backgiound corrected)

Analysis Date October 4, 1996

Log-in No. $\quad 97-00030$

Sample size $\quad 1.28 \mathrm{cc}$ stp

Liquid found in vial $0 \mathrm{ml}$

Room temperature $\quad .72 .5^{\circ} \mathrm{F} \quad$ Mole Estimate of

Percent Precision

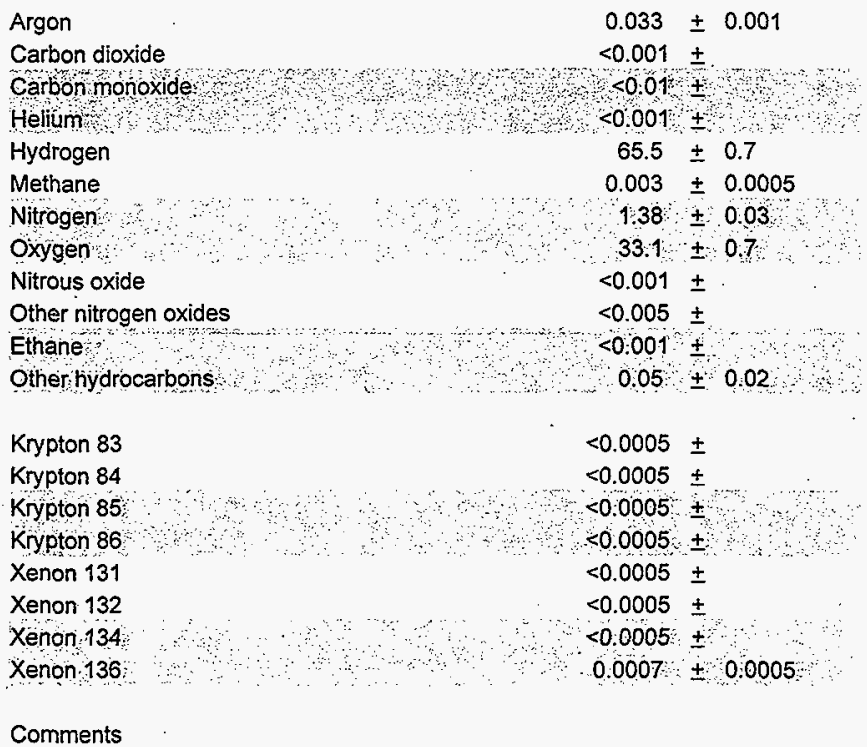




\section{Pacific Northwest National Laboratory}

From: $\quad 325$ Gas \& Isotopic Mass Spectrometry

Phone: $\quad$ (509) 376-3358/ mail slot P7-22

Date: $\quad$ October 08, 1996

Subject: Gas Species Analysis

To: $\quad$ D.J. Trimble

Analytical procedure: PNL-MA-599 ALO-284

Record book ACL $6 \quad$ Page 107

Measurement and test equipment WC38625

Sample Id. E-26 (Backgíound corrected)

Analysis Date October 4, 1996

Log-in No. $\quad 97-00031$

Sample size $\quad 1.27 \mathrm{cc}$ stp

Liquid found in vial $0.05 \mathrm{ml}$

Room temperature $\quad 70.0^{\circ} \mathrm{F}$ Mole Estimate of

Percent Precision

Argon

Carbon dioxide

$0.142 \pm 0.003$

Carbon monoxide

Helium

$<0.001 \pm$

Hydrogen

$<0.01 \pm$

Methane

$0.001 \pm$

Nitrogen

$57.6 \pm 0.6$

$0.004 \pm 0.0005$

Oxygen

$10.5 \pm 0.2$

Nitrous oxide

$317 \pm 0.4$

Other nitrogen oxides

$<0.001 \pm$

$<0.005 \pm$

Ethane $<0.001$

Other hydrocarbons

Krypton 83

$<0.0005 \pm$

Krypton 84

$<0.0005 \pm$

Krypton 85

$<0.0005$

$<0.0005$

Krypton 86

$<0.0005 \pm$

Xenon 131

$<0.0005 \pm$

Xenon 132

$<0.0005 \pm$

Xenon 134

$<0.0005 \div$

Xenon 136

Comments 


\section{Pacific Northwest National Laboratory}

From: $\quad 325$ Gas \& Isotopic Mass Spectrometry

Phone: $\quad$ (509) 376-3358/ mail slot P7-22

Date: $\quad$ October 08, 1996

Subject: Gas Species Analysis

To: $\quad$ D.J. Trimble

Analytical procedure: PNL-MA-599 ALO-284

Record book ACL $6 \quad$ Page 107

Measurement and test equipment WC38625

Sample ld. E-27 (Backgiound corrected)

Analysis Date October 4, 1996

Log-in No. $\quad 97-00032$

Sample size $\quad 1.28 \mathrm{cc} \mathrm{stp}$

Liquid found in vial $0.8 \mathrm{ml}$

Room temperature $\quad 70.7^{\circ} \mathrm{F} \quad$ Mole Estimate of

Percent Precision

Argon

$0.129 \pm 0.003$

Carbon dioxide $0.084 \pm 0.002$

Carbon monoxidè

$<0.01$

Helium $<0.001+$

Hydrogen

$61.8 \pm 0.7$

Methane

$0.005 \pm 0.0005$

Nitrogen

$119 \pm 0.2$

Oxygen

$26.1 \pm 0.5$

Nitrous oxide

$<0.01 \pm$

Other nitrogen oxides

$<0.005 \pm$

Ethane

$<0: 001$

Other hydrocarbons

$0.01 \pm 0.005$

Krypton 83

$<0.0005 \pm$

Krypton 84

Kiypton 85

Krypton 86

Xenon 131

$<0.0005 \pm$

Xenon 132

$<0,0005 \pm$

$<0.0005$

$0.001 \pm 0.0005$

$0.002 \pm 0.0005$

Xenon 134

$0.003+0.0005$

Xenon 136

$0.005 \pm 0.0005$

Comments 


\section{Pacific Northwest National Laboratory}

From: $\quad 325$ Gas \& Isotopic Mass Spectrometry

Phone: $\quad$ (509) 376-3358/mail slot P7-22

Date: $\quad$ October 08, 1996

Subject: Gas Species Analysis

To: $\quad$ D.J. Trimble

Analytical procedure: PNL-MA-599 ALO-284

Record book ACL $6 \quad$ Page 107

Measurement and test equipment . WC38625

Sample Id. E-28 (Backgiound corrected)

Analysis Date October 4, 1996

Log-in No. $\quad 97-00033$

Sample size $\quad 1.26 \mathrm{cc}$ stp

Liquid found in vial $1.0 \mathrm{ml}$

Room temperature $\quad 69.8^{\circ} \mathrm{F} \quad$ Mole Estimate of

Percent Precision

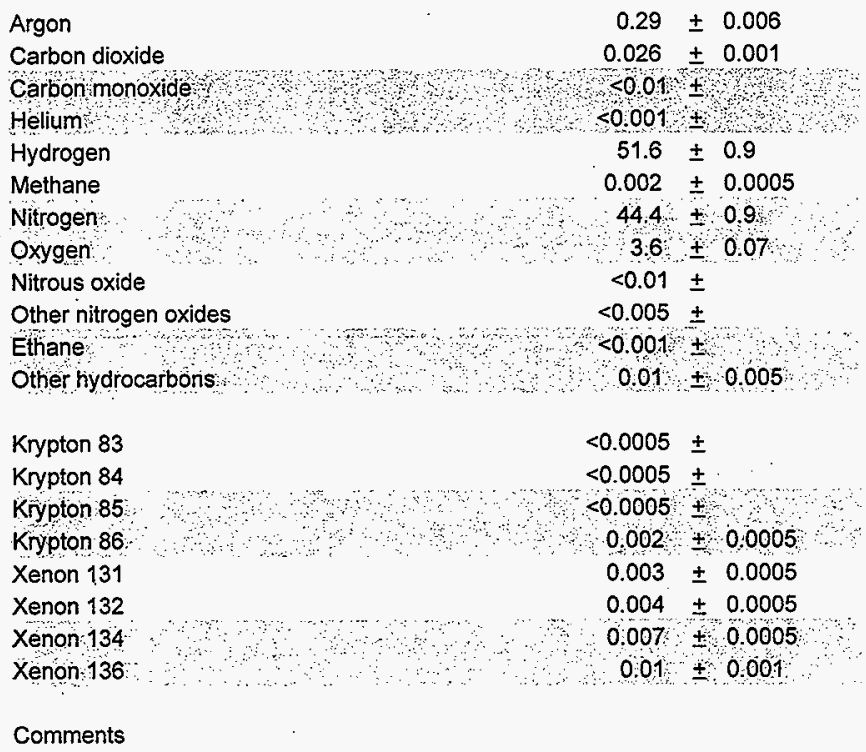




\section{Pacific Northwest National Laboratory}

From: $\quad 325$ Gas \& Isotopic Mass Spectrometry

Phone: $\quad$ (509) 376-3358 / mail slot P7-22

Date: $\quad$ October 08, 1996

Subject: Gas Species Analysis

To: $\quad$ D.J. Trimble

Analytical procedure: PNL-MA-599 ALO-284

Record book ACL 6 Page 107

Measurement and test equipment WC38625

Sample 1d. E-29 (Background corrected)

Analysis Date $\quad$ October 4, 1996

Log-in No. $\quad 97-00034$

Sample size $\quad 1.27 \mathrm{cc}$ stp

Liquid found in vial $1.0 \mathrm{ml}$

Room temperature $69.8^{\circ} \mathrm{F} \quad$ Mole Estimate of

Percent Precision

Argon

$0.31 \pm 0.006$

Carbon dioxide

$0.019 \pm 0.001$

Carbon monoxide $<0.01+$

Helium $<0.001$

Hydrogen

$50.2 \pm 0.9$

Methane

$0.002 \pm 0.0005$

Nitrogen

$482 \pm 0.9$

Oxygen

$1.23 \pm 0.02$

Nitrous oxide

$<0.01 \pm$

Other nitrogen oxides

$<0.005 \pm$

Ethane $<0,001 \pm$

Other hydrocarbons

Krypton 83

$<0.0005 \pm$

Krypton 84

$<0.0005 \pm$

Krypton 85

$<0.0005 \pm$

Krypton 86

$0.003+$

Xenon 131

Xenon 132

$+0.0005$

Xenon 134

$0.005 \pm 0.0005$

Xenon 136

$0.007 \pm 0.0005$

$0.011 \pm 0.001$

Comments 


\section{Pacific Northwest National Laboratory}

From: $\quad 325$ Gas \& Isotopic Mass Spectrometry

Phone: $\quad$ (509) 376-3358/mail slot P7-22

Date: $\quad$ October 08, 1996

Subject: $\quad$ Gas Species Analysis

To: D.J. Trimble

Analytical procedure: PNL-MA-599 ALO-284

Record book ACL $6 \quad$ Page 107

Measurement and test equipment WC38625

Sample ld. $\quad$ E-30 (Background corrected)

Analysis Date October 4, 1996

Log-in No. $\quad 97-00035$

Sample size $\quad 0.14$ cc stp

Liquid found in vial $0.8 \mathrm{ml}$

Room temperature $68.7^{\circ} \mathrm{F}$. Mole Estimate of

Percent Precision

\begin{tabular}{|c|c|c|}
\hline Argon & 0.76 & \pm 0.02 \\
\hline Carbon dioxide & $<0.01$ & \pm \\
\hline Carbon monoxide & $<001$ & \\
\hline Helium & $<0001$ & \pm \\
\hline Hydrogen & 26.2 & \pm 0.5 \\
\hline Methane & 0.024 & \pm 0.002 \\
\hline Nitrogen & 562 & \pm 0.5 \\
\hline Oxygen & 16.8 & \pm 0.3 \\
\hline Nitrous oxide & $<0.01$ & \pm \\
\hline Other nitrogen oxides & $<0.005$ & \pm \\
\hline Ethane: & $<0001$ & \pm \\
\hline Other hydrocarbons & 0.06 & \pm 0.03 \\
\hline Krypton 83 & $<0.0005$ & \pm \\
\hline Krypton 84 & $<0.0005$ & \pm \\
\hline Kryptôn 85 & $<0.0005$ & \pm \\
\hline Krypton 86 & $<0,0005$ & \pm \\
\hline Xenon 131 & $<0.0005$ & \pm \\
\hline Xenon 132 & $<0.0005$ & \pm \\
\hline Xenon 134: & $<00005$ & \pm \\
\hline Xenon 136 & $<0.0005$ & $\pm \cdots$ \\
\hline Comments & & \\
\hline
\end{tabular}




\section{Pacific Northwest National Laboratory}

From: $\quad 325$ Gas \& Isotopic Mass Spectrometry

Phone: $\quad$ (509) 376-3358 / mail slot P7-22

Date: $\quad$ October 08, 1996

Subject: Gas Species Analysis

To: D.J. Trimble

Analytical procedure: PNL-MA-599 ALO-284

Record book ACL $6 \quad$ Page 107

Measurement and test equipment WC38625

Sample Id. E-32 (Background corrected)

Analysis Date October 4, 1996

Log-in No. $\quad 97-00036$

Sample size $\quad 0.14 \mathrm{cc}$ stp

Liquid found in vial $0.8 \mathrm{ml}$

Room temperature $68.5^{\circ} \mathrm{F} \quad$ Mole Estimate of

Percent : Precision

Argon

$1.08 \pm 0.02$

Carbon dioxide

$0.101 \pm 0.002$

Carbon monoxide

$<0.011$

Héliúm

$<0,001$

Hydrogen

$0.54 \pm 0.01$

Methane

$0.072 \pm 0.001$

Nitrogen

$72 \pm 0.5$

oxygen

$26.2 \pm 0.5$

Nitrous oxide

$<0.01 \pm$

Other nitrogen oxides

Ethane

$<0.005 \pm$

$<0.001 \pm$

Other hydrocarbons

$$
0.06 \pm 0.03
$$

Krypton 83

$<0.0005 \pm$

Krypton 84

$<0.0005 \pm$

Krypton 85

$<0,0005 \pm$

Krypton 86

$<00005$

Xenon 131

$<0.0005 \pm$

Xenon 132

$<0.0005 \pm$

Xenon 134

$<0.0005$

Xenon 136

$<0.0005 \pm$

Comments 


\section{Pacific Northwest National Laboratory}

From: $\quad 325$ Gas \& Isotopic Mass Spectrometry

Phone: (509) 376-3358/mail slot P7-22

Date: $\quad$ October 14, 1996

Subject: Gas Species Analysis

To: $\quad$ D.J. Trimble

Analytical procedure: PNL-MA-599 ALO-284

Record book ACL $6 \quad$ Page 109

Measurement and test equipment WC38625

Sample ld. E-33 (Background Corrected)

Analysis Date October 11, 1996

Log-in No. $\quad 97-00099$

Sample size $\quad 1.27 \mathrm{cc} \mathrm{stp}$

Liquid found in vial $0.4 \mathrm{ml}$

Room temperature $\quad 68.2^{\circ} \mathrm{F} \quad$ Mole Estimate of

Percent Precision

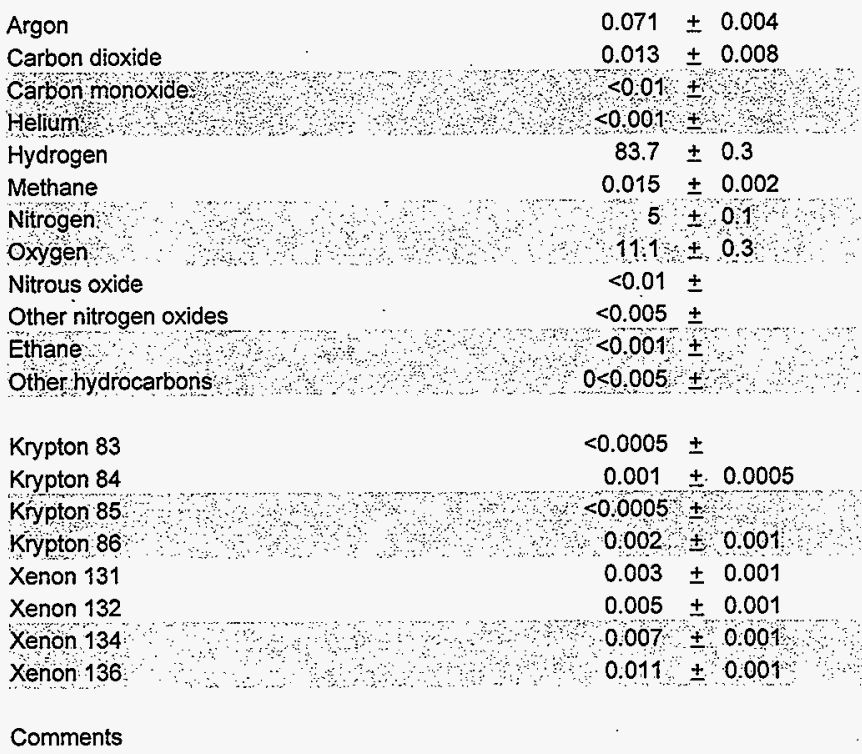




\section{Pacific Northwest National Laboratory}

From: $\quad 325$ Gas \& Isotopic Mass Spectrometry

Phone: $\quad$ (509) 376-3358/mail slot P7-22

Date: $\quad$ October 14, 1996

Subject: Gas Species Analysis

To: $\quad$ D.J. Trimble

Analytical procedure: PNL-MA-599 ALO-284

Record book ACL $6 \quad$ Page 109

Measurement and test equipment WC38625

Sample ld. E-34 (Background Corrected)

Analysis Date October 11, 1996

Log-in No. $\quad 97-00100$

Sample size $\quad 1.23 \mathrm{cc}$ stp

Liquid found in vial $3.8 \mathrm{ml}$

Room temperature $\quad 68.9^{\circ} \mathrm{F} \quad$ Mole Estimate of

Percent Precision

\begin{tabular}{|c|c|c|}
\hline Argon & 0.104 & \pm 0.004 \\
\hline Carbon dioxide & 0.06 & \pm 0.01 \\
\hline Carbon monoxide & $<<0,01$ & \pm \\
\hline Helium & $<0001$ & $\pm N-1$ \\
\hline Hydrogen & 81.4 & \pm 0.3 \\
\hline Methane & 0.032 & \pm 0.003 \\
\hline trogen ? & 59 & +01 \\
\hline Oxygen & 125 & +0.3 \\
\hline Nitrous oxide & $<0.01$ & \pm \\
\hline Other nitrogen oxides & $<0.005$ & \pm \\
\hline Ethane & $<0,001$ & \pm \\
\hline Other hydrocarbons & 0.01 & \pm 0.005 \\
\hline Krypton 83 & $<0.0005$ & \pm \\
\hline Krypton 84 & $<0.0005$ & \pm \\
\hline Krypton 85 & $<0.0005$ & \pm \\
\hline Krypton 86 & $<00005$ & \pm \\
\hline Xenon 131 & 0.002 & \pm 0.001 \\
\hline Xenon 132 & 0.003 & \pm 0.001 \\
\hline Xenon 134 & 0.004 & \pm 0.001 \\
\hline Xenon 136 & 0.006 & +0001 \\
\hline
\end{tabular}

Comments 


\section{Pacific Northwest National Laboratory}

From: $\quad 325$ Gas \& Isotopic Mass Spectrometry

Phone: (509) 376-3358/ mail slot P7-22

Date: $\quad$ October 14, 1996

Subject: Gas Species Analysis

To: $\quad$ D.J. Trimble

Analytical procedure: PNL-MA-599 ALO-284

Record book ACL 6 Page 109

Measurement and test equipment WC38625

Sample Id. $\quad$ E-34 Dup 2nd syringe (Background Corrected)

Analysis Date October 14,1996

Log-in No. $\quad 97-00100$

Sample size $\quad 1.13 \mathrm{ccstp}$

Liquid found in vial $3.8 \mathrm{ml}$

Room temperature $69.8^{\circ} \mathrm{F} \quad$ Mole Estimate of

Percent Precision

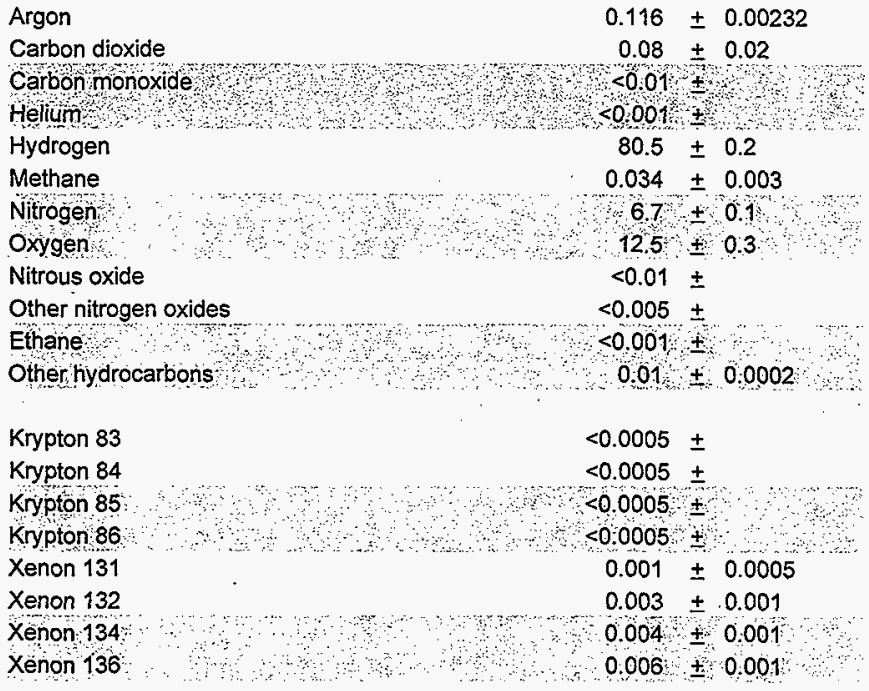

Comments 
From: $\quad 325$ Gas \& Isotopic Mass Spectrometry

Phone: $\quad$ (509) 376-3358 / mail slot P7-22

Date: October 14, 1996

Subject: Gas Species Analysis

To: $\quad$ D.J. Trimble

Analytical procedure: PNL-MA-599 ALO-284

Record book ACL $6 \quad$ Page 109

Measurement and test equipment WC38625

Sample Id. E-36 (Background Corrected)

Analysis Date October 11, 1996

Log-in No. 97-00101

Sample size $\quad 1.27$ cc stp

Liquid found in vial $0.1 \mathrm{ml}$

Room temperature $69.3^{\circ} \mathrm{F}$. Mole Estimate of

Percent . Precision

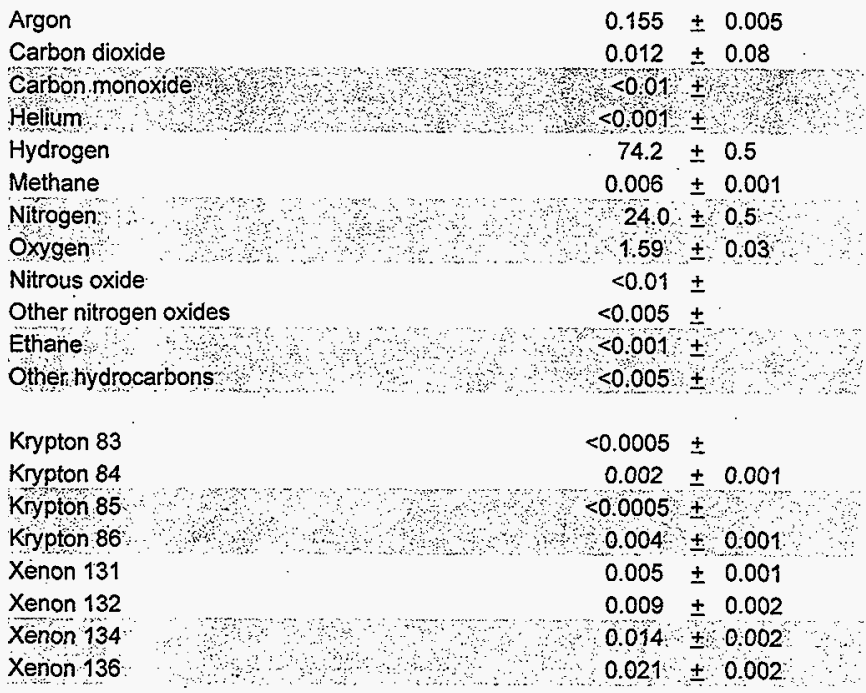

Comments 


\section{Pacific Northwest National Laboratory}

From: $\quad 325$ Gas \& Isotopic Mass Spectrometry

Phone: $\quad$ (509) 376-3358 / mail slot P7-22

Date: $\quad$ October 14, 1996

Subject: Gas Species Analysis

To: $\quad$ D.J. Trimble

Analytical procedure: PNL-MA-599 ALO-284

Record book ACL 6

Page 109

Measurement and test equipment WC38625

Sample 1d. $\quad$ E37 (Background Corrected)

Analysis Date October 11,1996

Log-in No.

97-00102

Sample size

1.26

Liquid found in vial $0.4 \mathrm{ml}$

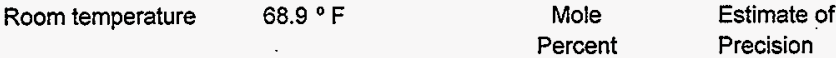

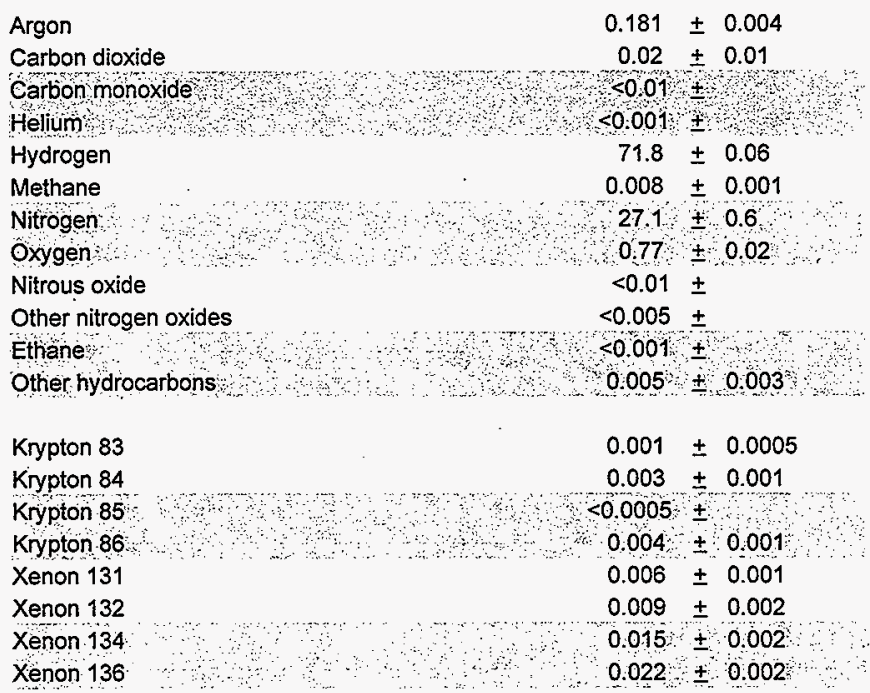

Comments 


\section{Pacific Northwest National Laboratory}

From: $\quad 325$ Gas \& isotopic Mass Spectrometry

Phone: $\quad$ (509) 376-3358/mail slot P7-22

Date: $\quad$ October 14, 1996

Subject: Gas Species Analysis

To: $\quad$ D.J. Trimble

Analytical procedure: PNL-MA-599 ALO-284

Record book ACL $6 \quad$ Page 109

Measurement and test equipment WC38625

Sample Id. E38 (Background Corrected)

Analysis Date October 11, 1996

Log-in No. $\quad 97-00103$

Sample size $\quad 1.28 \mathrm{cc} \mathrm{stp}$

Liquid found in vial $0.1 \mathrm{ml}$

Room temperature $68.5^{\circ} \mathrm{F} \quad$ Mole Estimate of

Percent Precision

\begin{tabular}{|c|c|c|}
\hline Argon & 0.206 & \pm 0.006 \\
\hline Carbon dioxide & $<0.001$ & \pm \\
\hline Carbon monoxide & $<0,01$ & + \\
\hline Heliự & $<0.001$ & $+6-1$ \\
\hline Hydrogen & 55 & \pm 1 \\
\hline Methane & 0.003 & \pm 0.001 \\
\hline Nitrogen & 44 & \pm 1 \\
\hline Oxygen & 0.63 & \pm 0.03 \\
\hline Nitrous oxide & $<0.01$ & \pm \\
\hline Other nitrogen oxides & $<0.005$ & \pm \\
\hline Ethane & $<0.001$ & \pm \\
\hline Other hydrocarbons & 0,005 & \pm 0003 \\
\hline Krypton 83 & 0.001 & \pm 0.0005 \\
\hline Krypton 84 & 0.002 & \pm 0.001 \\
\hline Krypton 85 & $<00005$ & \pm \\
\hline Krypton 86 & 0.003 & \pm 0.001 \\
\hline Xenon 131 & 0.004 & \pm 0.001 \\
\hline Xenon 132 & 0.007 & \pm 0.002 \\
\hline Xenon 134 & 0.012 & \pm 0.002 \\
\hline Xenon 136 & 0.018 & $\mp 0.002$ \\
\hline
\end{tabular}




\section{Pacific Northwest National Laboratory}

From: $\quad 325$ Gas \& Isotopic Mass Spectrometry

Phone: $\quad$ (509) 376-3358/mail slot P7-22

Date: $\quad$ October 14, 1996

Subject: Gas Species Analysis

To: $\quad$ D.J. Trimble

Analytical procedure: PNL-MA-599 ALO-284

Record book ACL $6 \quad$ Page 109

Measurement and test equipment WC38625

\begin{tabular}{|c|c|}
\hline Sample ld. & E39 (Background Corrected) \\
\hline Analysis Date & October 11, 1996 \\
\hline Log-in No. & $97-00104$ \\
\hline Sample size & $1.25 \mathrm{cc} \mathrm{stp}$ \\
\hline $\begin{array}{l}\text { Liquid found in vial } \\
\text { Room temperature }\end{array}$ & $\begin{array}{l}1.4 \mathrm{ml} \\
\quad 68.0^{\circ} \mathrm{F}\end{array}$ \\
\hline
\end{tabular}

Mole Estimate of

Percent Precision

\begin{tabular}{|c|c|c|}
\hline Argon & 0.135 & \pm 0.004 \\
\hline Carbon dioxide & 0.04 & \pm 0.01 \\
\hline Carbon monoxide & $<0.01$ & $+\mathrm{s}^{2}$ \\
\hline Heliumm & $<0.001$ & +4 \\
\hline Hydrogen & 82.1 & \pm 0.3 \\
\hline Methane & 0.044 & \pm 0.004 \\
\hline Nifirogen & 136 & +0.3 \\
\hline Oxygen & 405 & \pm 0.09 \\
\hline Nitrous oxide & $<0.01$ & \pm \\
\hline Other nitrogen oxides & $<0.005$ & \pm \\
\hline Ethane & 80.001 & \pm \\
\hline Other hydrocarbo & 0.005 & \pm 0.003 \\
\hline Krypton 83 & $<0.0005$ & \pm \\
\hline Krypton 84 & 0.002 & \pm 0.001 \\
\hline Kypton 85 & $<0.0005$ & +4 \\
\hline Koypton 86 & 0,003 & $\$ 0001$ \\
\hline Xenon 131 & 0.005 & \pm 0.001 \\
\hline Xenon 132 & 0.008 & \pm 0.002 \\
\hline Xenon 134 & 0.013 & \pm 0.002 \\
\hline Senon 136: & 0.020 & \pm 0002 \\
\hline
\end{tabular}

Comments 


\section{Pacific Northwest National Laboratory}

From: $\quad 325$ Gas \& Isotopic Mass Spectrometry

Phone: $\quad$ (509) 376-3358/mail slot P7-22

Date: $\quad$ October 22, 1996

Subject: Gas Species Analysis

To: $\quad$ D.J. Trimble

Analytical procedure: PNL-MA-599 ALO-284

Record book ACL 6 Page 110

Measurement and test equipment WC38625

Sample id.

E40 (Background Corrected)

Analysis Date $\quad$ October 18, 1996

Log-in No. $\quad 97-00181$

Sample size $\quad 3.24$ cc stp

Liquid found in vial $1.0 \mathrm{ml}$

Room temperature $\quad 71.8^{\circ} \mathrm{F} \quad$ Mole Estimate of

Percent Precision

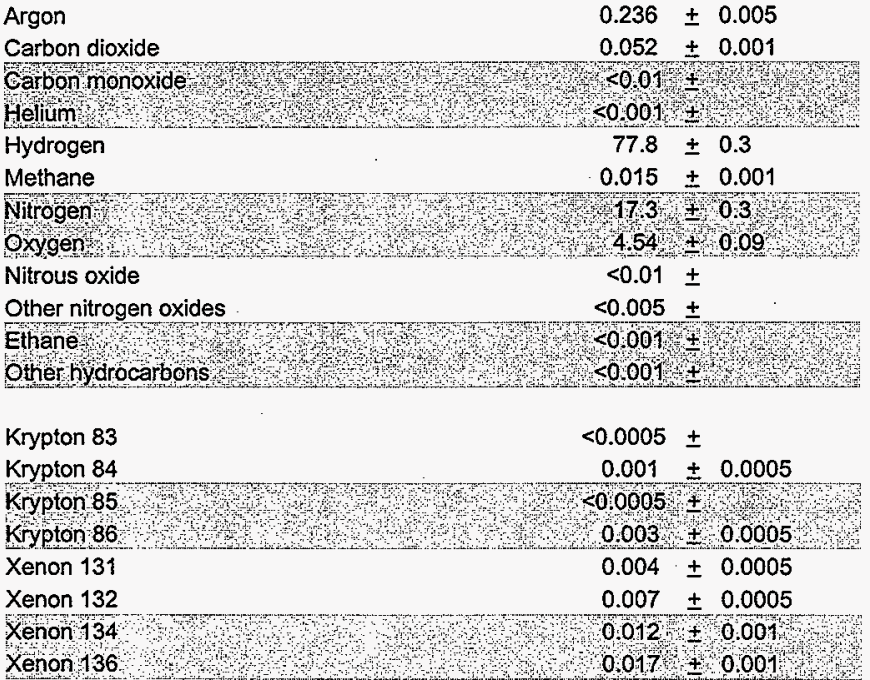

Comments 


\section{Pacific Northwest National Laboratory}

From: $\quad 325$ Gas \& Isotopic Mass Spectrometry

Phone: (509) 376-3358/ mail slot P7-22

Date: $\quad$ October 22, 1996

Subject: Gas Species Analysis

To:

D.J. Trimble

Analytical procedure: PNL-MA-599 ALO-284

Record book ACL 6

Page 110

Measurement and test equipment WC38625

Sample Id.

E41 (Background Corrected)

Analysis Date

October 18,1996

Log-in No.

97-00182

Sample size

$1.27 \mathrm{cc}$ stp

Liquid found in vial $2.6 \mathrm{ml}$

Room temperature $\quad 72.1^{\circ} \mathrm{F}$

Estimate of

Percent

Precision

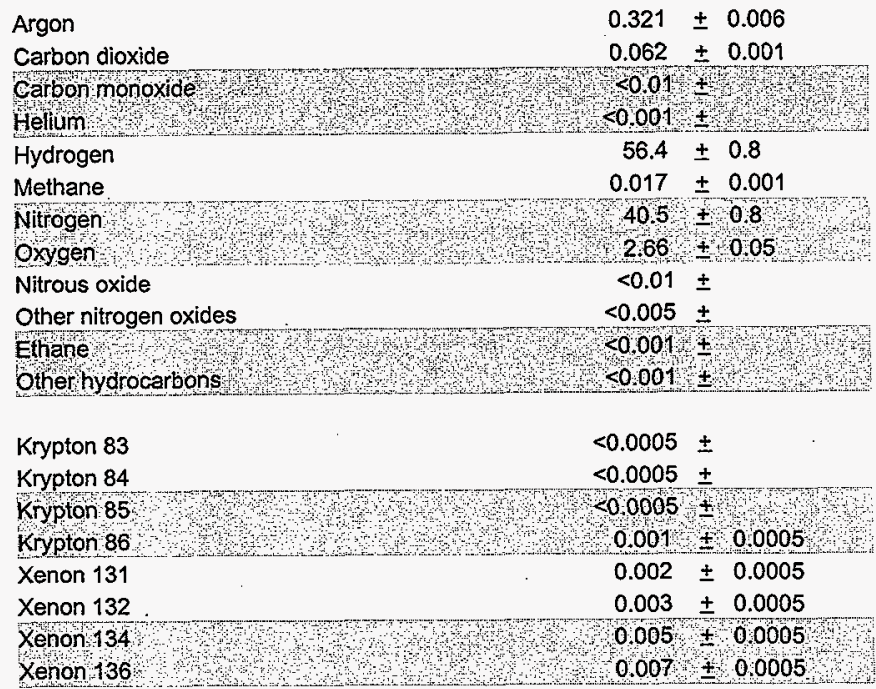

Comments 


\section{Pacific Northwest National Laboratory}

From: $\quad 325$ Gas \& Isotopic Mass Spectrometry

Phone: $\quad$ (509) 376-3358/ mail slot P7-22

Date: $\quad$ October 14, 1996

Subject: Gas Species Analysis

To: $\quad$ D.J. Trimble

Analytical procedure: PNL-MA-599 ALO-284

Record book ACL $6 \quad$ Page 109

Measurement and test equipment WC38625

Sample Id. E42 (Background Corrected)

Analysis Date October 11, 1996

Log-in No. $\quad 97-00105$

Sample size $\quad 1.21 \mathrm{cc}$ stp

Liquid found in vial $1.2 \mathrm{ml}$

Room temperature $\quad 67.8^{\circ} \mathrm{F}$

Mole Estimate of

Percent Precision

Argon

$0.167 \pm 0.00334$

Carbon dioxide $<0.005 \pm$

Carbon monoxide $<001 \pm$

Hêljum $<0001 \pm$

Hydrogen

$81.4 \pm 1.628$

Methane

$0.006 \pm 0.00012$

Nitrogen

$174 \pm 0.348$

oxygen

$0.99 \pm 0.0198$

Nitrous oxide

$<0.01 \pm$

Other nitrogen oxides

$<0.005 \pm$

Ethane

Other hydrocarbons $<0.001 \pm$ $<0005 \pm$

Krypton 83

$<0.0005 \pm$

Krypton 84

$0.002 \pm 0.0005$

Krypton 85

$<0.0005$

Krypton 86

$0.003+0.001$

Xenon 131

$0.004 \pm 0.001$

Xenon 132

$0.008 \pm 0.002$

Xenon 134

$0.013+0.002$

Xenon 136

$0.020 \mp 0.002$

Comments 


\section{Pacific Northwest National Laboratory}

From: $\quad 325$ Gas \& Isotopic Mass Spectrometry

Phone: $\quad$ (509) 376-3358/ mail siot P7-22

Date: $\quad$ September 24, 1996

Subject: Gas Species Analysis

To: $\quad$ D.J. Trimble

Analytical procedure: PNL-MA-599 ALO-284

Record book ACL $6 \quad$ Page 104

Measurement and test equipment WC38625

Sample ld. E-49 (Background corrected)

Analysis Date September 20, 1996

Log-in No. $\quad 96-06757$

Sample size $\quad 0.87$ cc stp

Liquid found in vial $0 \mathrm{ml}$

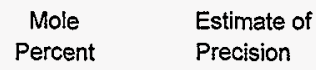

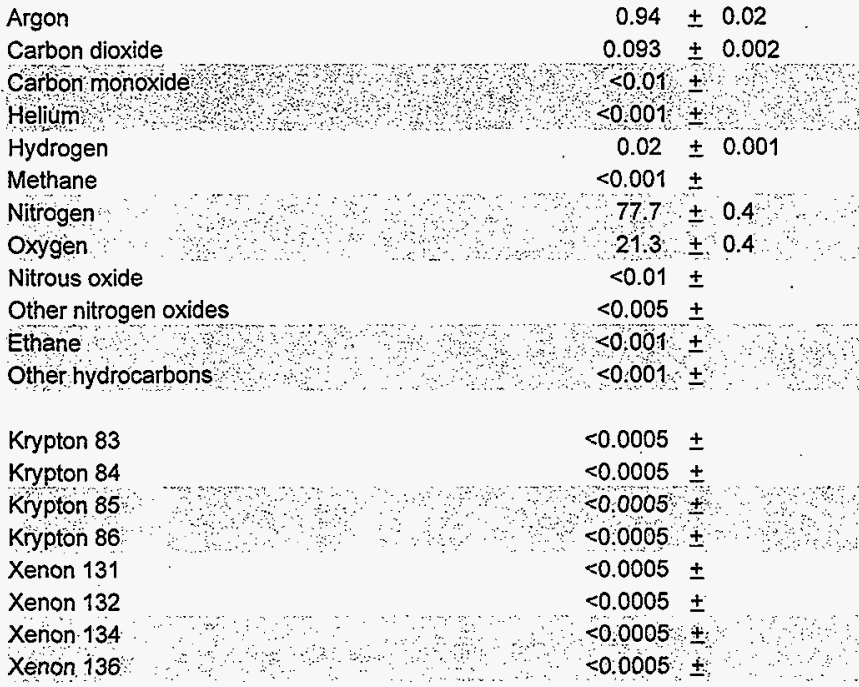

Comments Results corrected by subtracting out gas found in new vials 


\section{Pacific Northwest National Laboratory}

From: $\quad 325$ Gas \& Isotopic Mass Spectrometry

Phone: (509) 376-3358/ mail slot P7-22

Date: $\quad$ September 24, 1996

Subject: Gas Species Analysis

To: $\quad$ D.J. Trimble

Analytical procedure: PNL-MA-599 ALO-284

Record book ACL $6 \quad$ Page 105

Measurement and test equipment WC38625

Sample 1d. E-49 dup (Background corrected)

Analysis Date September 20,1996

Log-in No. $\quad 96-06757$

Sample size $\quad 0.82$ cc stp

Liquid found in vial $0 \mathrm{ml} \quad$ Mole Estimate of

Percent Precision

\begin{tabular}{|c|c|c|}
\hline Argon & 0.94 & \pm 0.02 \\
\hline Carbon dioxide & 0.092 & \pm 0.002 \\
\hline Carbon monoxide & $<0.01$ & \pm \\
\hline Helium & $<0.001$ & \pm \\
\hline Hydrogen & 0.018 & \pm 0.001 \\
\hline Methane & $<0.001$ & \pm \\
\hline Nitrogen & 777 & \pm 0.4 \\
\hline Oxygen: & 21.3 & \pm 0.4 \\
\hline Nitrous oxide & $<0.01$ & \pm \\
\hline Other nitrogen oxides & $<0.005$ & \pm \\
\hline Ethane & $<0.001$ & \pm \\
\hline Other hydrocarbons & $<0.001$ & \pm \\
\hline Krypton 83 & $<0.0005$ & \pm \\
\hline Krypton 84 & $<0.0005$ & \pm \\
\hline Krypton 85 & $<0.0005$ & \pm \\
\hline Krypton 86 & $<0.0005$ & \pm \\
\hline Xenon 131 & $<0.0005$ & \pm \\
\hline Xenon 132 & $<0.0005$ & \pm \\
\hline Xenon 134 & $<0.0005$ & \pm \\
\hline Xenon 136 & $<0.0005$ & \pm \\
\hline
\end{tabular}




\begin{tabular}{|c|c|c|c|c|c|c|}
\hline \multicolumn{7}{|c|}{ DISTRIBUTION SHEET } \\
\hline To & \multirow{2}{*}{\multicolumn{3}{|c|}{$\begin{array}{l}\text { From } \\
\text { Spent Nuclear Fuel Evaluations } \\
\text { 2T650 }\end{array}$}} & \multicolumn{3}{|c|}{ Page 1 of 2} \\
\hline Distribution & & & & \multicolumn{3}{|c|}{ Date $03 / 03 / 97$} \\
\hline \multicolumn{4}{|l|}{ Project Title/Work Order } & \multicolumn{3}{|c|}{ EDT No. 620782} \\
\hline Spent Nuclear Fuel Pro. & D-SNF-TI-049, & ev. 0 & & \multicolumn{3}{|c|}{ ECN No. } \\
\hline Name & MSIN & $\begin{array}{l}\text { Text } \\
\text { With All } \\
\text { Attach. }\end{array}$ & Text O & & $\begin{array}{l}\text { Attach./ } \\
\text { Appendix } \\
\text { Only }\end{array}$ & $\begin{array}{c}\text { EDT/ECN } \\
\text { Only }\end{array}$ \\
\hline
\end{tabular}

U.S. Department of Energy,

Richland Operations Office

E. D. MacAlister

J. Shuen

S7-41

$57-41$

$\underline{x}$

B\&w Protec, Inc.

T. L. Welsh

T4-40

$X$

Fluor Daniel Northwest

M. Kummerer

A3-34 $X$

Pacific Northwest National Laboratory

J. Abrefah

S. C. Marschman

P7-27 $X$

P7-27 $X$

SEG

S. H. Peck

R3-85

$\mathrm{X}$

SGN Eurisys Services Corporation

A. L. Pajunen

R3-86

S3-90

$x$

E. F. Riedel

WSTRN

N. R. Roe

HO-40

$x$

Duke Engineering \& Services Northwest, Inc.

C. J. Alderman

R. B. Baker

D. W. Bergmann

K. H. Bergsman

A. E. Bridges

L. D. Bruggeman

S. P. Burke

B. S. Carlisle

S. A. Chastain

R. G. Cowan

C. Defigh-Price

D. R. Duncan

R3-48

$\mathrm{HO}-40$

R3-86

R3-48

$\mathrm{HO}-40$

$\mathrm{H} 0-40$

$\times 3-56$

R3-85

$\mathrm{HO}-40$

R3-86

X3-79

R3-86

$x$
$x$
$x$
$x$
$x$
$x$
$x$
$x$
$x$
$x$
$x$
$x$ 


\begin{tabular}{|c|c|c|c|c|c|c|}
\hline \multicolumn{7}{|c|}{ DISTRIBUTION SHEET } \\
\hline To & \multirow{2}{*}{\multicolumn{3}{|c|}{$\begin{array}{l}\text { From } \\
\text { Spent Nuclear Fuel Evaluations } \\
2 T 650\end{array}$}} & \multicolumn{3}{|c|}{ Page 2 of 2} \\
\hline Distribution & & & & \multicolumn{3}{|c|}{ Date 03/03/97 } \\
\hline \multicolumn{4}{|l|}{ Project Title/Work Order } & \multicolumn{3}{|c|}{ EDT No. 620782} \\
\hline Spent Nuclear Fuel Pro & D-SNF-TI-049, & ev. 0 & & \multicolumn{3}{|c|}{ ECN No. } \\
\hline Name & MSIN & $\begin{array}{l}\text { Text } \\
\text { With All } \\
\text { Attach. }\end{array}$ & Text $\mathrm{O}$ & & $\begin{array}{l}\text { Attach./ } \\
\text { Appendix } \\
\text { Only }\end{array}$ & $\begin{array}{c}\text { EDT/ECN } \\
\text { Only }\end{array}$ \\
\hline
\end{tabular}

\section{Duke Engineering \& Services, Hanford Inc.}

J. R. Frederickson

R. G. Gant

L. H. Goldman

M. A. Green

S. L. Hecht

F. G. Hudson

G. S. Hunacek

J. J. Jernberg

A. T. Kee

C. D. Kirk

R. E. Lacey

M. J. Langevin

L. A. Lawrence

P. G. LeRoy

C. D. Lucas

P. J. MacFarlan

B. J. Makenas

R. H. Meichle

W. C. Mills

R. P. Omberg

T. R. Pauly

K. L. Pearce

C. C. Pitkoff

A. L. Pitner

D. L. Sherrel1

D. W. Siddoway

D. W. Smith

J. A. Swenson

D. S. Takasumi

C. A. Thompson

D. J. Trimble

M. J. Wiemers

SNF Project Files

Central Files

Fluor Daniel Hanford, Inc.

E. W. Gerber

Numatec Hanford Corporation

C. R. Miska

W. L. Willis

$\begin{array}{ll}\text { R3-86 } & X \\ \text { X3-79 } & X \\ \text { R3-86 } & X \\ \text { X3-56 } & X \\ \text { H0-40 } & X \\ \text { R3-11 } & X \\ \text { X3-79 } & X \\ \text { X3-72 } & X \\ \text { R3-86 } & X \\ \text { R3-85 } & X \\ \text { R3-15 } & X \\ \text { X3-74 } & X \\ \text { H0-40 } & X \\ \text { R3-15 } & X \\ \text { X3-67 } & X \\ \text { H0-40 } & X \\ \text { H0-40 } & X \\ \text { X3-79 } & X \\ \text { R3-85 } & X \\ H 0-40 & X \\ \text { X3-85 } & X \\ \text { R3-48 } & X \\ \text { X3-85 } & X \\ \text { H0-40 } & X \\ \text { R3-86 } & X \\ \text { X3-71 } & X \\ \text { R3-15 } & X \\ \text { R3-11 } & X \\ \text { X3-85 } & X \\ \text { R3-85 } & X \\ \text { H0-40 } & X \\ \text { X3-85 } & X \\ \text { R3-11 } & X \\ \text { A3-88 } & X \\ & \end{array}$

R3-11 X

R3-86

R3-86
$X$
$X$
$X$
$X$
$X$
$X$
$X$
$X$
$X$
$X$
$X$
$X$
$X$
$X$
$X$
$X$
$X$
$X$
$X$
$X$
$X$
$X$
$X$
$X$
$X$
$X$
$X$
$X$
$X$
$X$
$X$
$X$
$X$ 